\title{
Numerical modeling transient processes in a long gas pipeline
}

\author{
Chekurin V. F. ${ }^{1,2}$, Khymko O. M. ${ }^{3}$ \\ ${ }^{1}$ Pidstryhach Institute for Applied Problems of Mechanics and Mathematics, \\ National Academy of Sciences of Ukraine, \\ 3-b Naukova Str., 79060, Lviv, Ukraine \\ ${ }^{2}$ Kujawy and Pomorze University in Bydgoszcz, \\ 55-57 Torunska Str., 85-023, Bydgoszcz, Poland \\ ${ }^{3}$ Lviv Polytechnic National University, \\ 12 Bandera Str., 79013, Lviv, Ukraine
}

(Received 7 Septemder 2019; Revised 26 Septemder 2019; Accepted 7 October 2019)

\begin{abstract}
Unsteady processes of isothermal natural gas flow, emerging in a long pipeline when there is switching-over from one stationary process to another stationary process, have been considered in this paper. The one-dimensional system of gas dynamics equations is used for that purpose. It includes equations for conservation of mass and momentum written relative to of dimensionless mass and flow densities. Three boundary-value problems formulated for this system define three models for control of the transient processes. The problems differ by the boundary (control) functions imposed at the ends of the gas pipeline. A unified model for the control functions is introduced. According to this model, such a function is defined by four real parameters. That restricts the class of control functions by the smooth ones monotonically varying from the value characteristic for the first stationary regime to the other one specific for the second stationary regime. The transient processes realized with the use of the models for various values of control parameters are analyzed numerically in this paper. Application of the considered mathematical models and obtained results of conducted case-studies for planning the transient regimes of pipelines operation are discussed in the paper.
\end{abstract}

Keywords: gas dynamics, long pipeline, transient processes.

2000 MSC: $76 \mathrm{D} 55$

UDC: 533:519.6:621.64.029

DOI: $10.23939 / \mathrm{mmc} 2019.02 .220$

\section{Introduction}

Main gas pipelines are very effective for natural gas transmission on large distances [1]. Optimally they operate in stationary regimes. But non-stationary processes are also used in the operation of these gas transportation facilities.

Unsteady gas flows in a long pipeline were considered by many authors with various purposes, in particular, to examine approximations of gas state equations and their impact on solutions of nonstationary problems $[2,3]$, to clear up the role of thermal processes in pipeline gas dynamics [4,5], to describe propagation of small disturbances of pressure in the gas flowing in the pipeline [6-8], to study transient processes emerging at a local depressurization of the pipe $[9,10]$.

Unsteady processes are more often used in the operation of a gas pipeline to switch-over it from an actual stationary regime to another prescribed one. With this aim, one should increase or decrease the mechanical power transferring to the flow, changing operation regimes of compressor stations. That causes variations of the pressure and flow rate at the inflow and outflow of each pipeline section between two compressor stations. The transient process emerging at that in the section will depend on parameters being controlled at the section's inflow and outflow (pressure or flow rate), and on the time dependences of the controlled parameters. 
There are many ways to transfer the gas pipeline from one to another stationary regime. The aim of the paper is to study numerically how the algorithm of controlling by the transient process impacts this process's parameters.

\section{Models for control functions of transient processes}

We consider a section of a gas main as a long pipeline. Its inner diameter is $D_{\text {pipe }}$, the length is $L$. We suppose the profile of the pipeline elevation $H(x)$ and the coefficient of pipeline hydraulic resistance $\lambda(x)$ are known functions of coordinate $x$ along the pipeline trace. As well, we will assume that gas composition and physical-chemical property of all its components, needed for calculation the gas thermodynamic properties, are also known. In isothermal approximation, the movement of the gas in the pipeline is governing by two partial differential equations (PDE), describing the conservation of mass and momentum, as well as the thermal equation of state [6]. In dimensionless variables, these equations can be written as

$$
\begin{aligned}
\frac{\partial \rho}{\partial \tau} & =-M a \cdot \frac{\partial \rho v}{\partial \xi} \\
\frac{\partial \rho v}{\partial \tau} & =-M a \cdot \frac{\partial}{\partial \xi}\left(\rho v^{2}+\frac{Z_{t}}{M a^{2}} p\right)-M a \cdot \rho \frac{d \gamma}{d \xi}-M a \cdot \beta|v| v \rho \\
p & =z \theta \rho .
\end{aligned}
$$

Here $\tau \equiv t / t_{t}$ and $\xi \equiv x / L$ stand for the dimensionless time variable and coordinate along the pipeline trace, $\rho \equiv D / D_{t}, p \equiv P / P_{t}$ and $v \equiv V / V_{t}$, are dimensionless gas parameters: density, pressure and velocity correspondingly, $D_{t}, P_{t}$ and $V_{t}$ are typical for this pipeline value of corresponding dimensional gas parameters: density $D$, pressure $P$ and velocity $V ; \theta$ is dimensionless temperature (we put $\theta \equiv 1$ in considered here isothermal approximation $T=$ const); $z \equiv Z / Z_{t}$ stands for normalized compressibility factor of the gas, where $Z_{t}=Z_{t}\left(D_{t}, T\right)$ is the value of the compressibility factor $Z=Z(D, T)$, calculated at $D_{t}$ and $T ; M a \equiv V_{t} / C_{t}$ is the Mach number, where $C_{t}$ is typical sonic velocity, defined from thermal equation of state $P=Z(D, T) R_{g} T D$ as $C_{t}^{2}=\left.(\partial P / \partial D)\right|_{Z=1, D=D_{t}}=R_{g} T$, where $R_{g} \equiv R / \mu_{g}, R$ is universal gas constant, $\mu_{g}$ is molar mass of the gas. Parameters $\beta=\beta(\xi), \gamma=\gamma(\xi)$ in (2) are defined as

$$
\gamma(x / L)=\frac{g H(x)}{V_{t}^{2}}, \quad \beta(x / L)=\frac{\lambda(x) L}{2 D_{\text {pipe }}},
$$

where $g$ is the gravity acceleration.

Equations (1) and (2) contain three dependent variables: $\rho(\xi, \tau), p(\xi, \tau)$ and $v(\xi, \tau)$. Applying relation (3), we can reduce them to the closed system of two PDF for key functions $\rho(\xi, \tau)$ and $j(\xi, \tau)$ :

$$
\begin{array}{ll}
\operatorname{PDE}_{\rho}: & \frac{\partial \rho}{\partial \tau}=-M a \cdot \frac{\partial j}{\partial \xi} \\
\operatorname{PDE}_{j}: & \frac{\partial j}{\partial \tau}=-M a \cdot \frac{\partial}{\partial \xi}\left(j^{2} \rho^{-1}+\frac{Z}{M a^{2}} \rho\right)-M a \cdot \rho \frac{d \gamma}{d \xi}-M a \cdot \beta|j| j \rho^{-1},
\end{array}
$$

where $j \equiv J / J_{t}=\rho v$ is dimensionless density of mass flow, $J_{t}$ is the typical value of mass flow density $J=\rho V$.

As we can see, thanks to such choice of the key functions, the first equation of the system (5) appears linear.

In stationary case, when $\partial \rho / \partial \tau=\partial j / \partial \tau \equiv 0$, from the first equation (5) we obtain $\partial j / \partial \xi=0 \rightarrow$ $j=$ const, and the second one leads to first order non-linear ordinary differential equation (ODE) for 
stationary density distribution $\rho(\xi)$ in the pipeline:

$$
\mathrm{ODE}_{\rho}: \quad \frac{d \rho}{d \xi}=-\frac{\beta \rho j^{2}+\rho^{3} \frac{\partial \gamma}{\partial \xi}}{j^{2}+\frac{Z_{t} \rho^{2}}{M a^{2}}\left(z+\rho \frac{\partial z}{\partial \rho}\right)},
$$

To determine density distribution $\rho(\xi)$ in the pipeline in stationary state, one should define a value of dimensionless density of mass flow $j$, and then formulate and solve an initial value problem (IVP) for ODE (6). Two such IVP can be considered - in the first one the initial condition (IC) is prescribed on the left end $\xi=0$ of the segment and, in the second one - on the right segment's end $\xi=1$ :

$$
\mathrm{IC}_{1}:\left.\quad \rho\right|_{\xi=0}=\rho^{\text {in }}, \quad \mathrm{IC} \mathrm{C}_{2}:\left.\quad \rho\right|_{\xi=2}=\rho^{\text {out }},
$$

where $\rho^{\text {in }}$ and $\rho^{\text {out }}$ are given real values.

The problems $\mathrm{IVP}_{1}=\left\{\mathrm{ODE}_{\rho}, \mathrm{IC}_{1}\right\}$ and $\mathrm{IVP}_{2}=\left\{\mathrm{ODE}_{\rho}, \mathrm{IC}_{2}\right\}$ represent two models for control by the stationary flow process. Their sets of control parameters are $\Pi_{\mathrm{I}}=\left\{j, \rho^{i n}\right\}$ and $\Pi_{\mathrm{II}}=\left\{j, \rho^{\text {in }}\right\}$ correspondingly. This means that, choosing, for instance, model IVP $\mathrm{I}$ and prescribing a values for both elements of set $\Pi_{\mathrm{I}}$, we fully determine stationary flow in the pipeline.

To determine the functions $\rho(\xi, \tau)$ and $j(\xi, \tau)$ of a transient process Transient ${ }_{1-2}$ one should first determine two stationary $\operatorname{processes}$ Stationar $_{1}$ and Stationar 2 . Here Stationar ${ }_{1}$ is the starting for Transient $_{1-2}$ stationary process, whereas Stationar 2 is the target stationary process for it. "Determine a stationary process" means here that one should choose a model $\left(\right.$ IVP $_{\text {I }}$ or IVPII) and define values for elements of corresponding set $\left(\Pi_{\mathrm{I}}\right.$ or $\left.\Pi_{\mathrm{II}}\right)$ of control parameters. Then one should formulate a boundary problem (BVP) for system (5). For that, it is necessary to define boundary conditions $(\mathrm{BC})$ and initial conditions for the system.

Since system (5) is of hyperbolic type, we should prescribe two boundary and two initial values for it. We will consider the next $\mathrm{BC}$ for system (5)

$$
\begin{array}{rll}
\mathrm{BC}_{\mathrm{I}}: & \left.\rho\right|_{\xi=0}=\rho^{\text {in }}(\tau), & \left.\rho\right|_{\xi=1}=\rho^{\text {out }}(\tau), \\
\mathrm{BC}_{\mathrm{II}}: & \left.\rho\right|_{\xi=0}=\rho^{\text {in }}(\tau), & \left.j\right|_{\xi=1}=j^{\text {out }}(\tau), \\
\mathrm{BC}_{\mathrm{III}}: & \left.j\right|_{\xi=0}=j^{\text {in }}(\tau), & \left.\rho\right|_{\xi=1}=\rho^{\text {out }}(\tau) .
\end{array}
$$

Here $\rho^{\text {in }}(\tau), \rho^{\text {out }}(\tau), j^{\text {in }}(\tau)$ and $j^{\text {out }}(\tau)$ are prescribed functions.

The initial conditions for system (5) we take in the form

$$
\text { IC }:\left.\quad \rho\right|_{\tau=0}=\rho_{1}(\xi),\left.\quad j\right|_{\tau=0}=j_{1} .
$$

Here $j_{1}$ is the value of dimensionless flow density for stationary process $\operatorname{Stationar}_{1}, \rho_{1}(\xi)$ is dimensionless density distribution in this process. Function $\rho_{1}(\xi)$ is the solution of $\operatorname{IVP}_{\mathrm{I}}$ or IVP II (depending on the used model for control by the stationary process).

With this, we can define three boundary-value problems:

$$
\begin{aligned}
\mathrm{BVP}_{\mathrm{I}} & =\left\{\mathrm{PDE}_{\rho}, \mathrm{PDE}_{j}, \mathrm{BC}_{\mathrm{I}}, \mathrm{IC}\right\} \\
\mathrm{BVP}_{\mathrm{II}} & =\left\{\mathrm{PDE}_{\rho}, \mathrm{PDE}_{j}, \mathrm{BC}_{\mathrm{II}}, \mathrm{IC}\right\} \\
\mathrm{BVP}_{\mathrm{III}} & =\left\{\mathrm{PDE}_{\rho}, \mathrm{PDE}_{j}, \mathrm{BC}_{\mathrm{II}}, \mathrm{IC}\right\}
\end{aligned}
$$

Boundary-value problems (10) determine tree models for control by transient process Transient ${ }_{1-2}$. Functions $\rho^{\text {in }}(\tau), \rho^{\text {out }}(\tau), j^{\text {in }}(\tau)$ and $j^{\text {out }}(\tau)$ of boundary conditions (8) are external or control functions. Defining a pair of them specific for a model BVP $\mathrm{K}_{\mathrm{K}}, \mathrm{K} \in\{\mathrm{I}, \mathrm{II}, \mathrm{III}\}$, we determine an algorithm for control by the transient process. Defining, for instance, the functions $\rho^{\text {in }}(\tau)$ and $\rho^{\text {out }}(\tau)$, we determine the algorithm for control by the transient process in correspondence with the model BVP ${ }_{\mathrm{I}}$. 


\section{Models for control functions of transient processes}

Four control functions of transient processes $\psi^{\text {in/out }} \in\left\{\rho^{\text {in/out }}, j^{\text {in/out }}\right\}$ should satisfy some conditions. The first condition is continuity requirement. It follows from the nature of compressor stations which are very inertial systems. Hence the pressure and flow rate at their inputs and outputs cannot change rapidly.

Other conditions follow from the requirement of compatibility of the control functions of process Transient $_{1-2}$ with the control parameters of both stationary processes Stationar ${ }_{1}$ and Stationar 2 . These conditions look like

$$
\begin{aligned}
& \left.\rho^{i n}\right|_{\tau=\tau_{\text {start }}^{\rho^{i n}}}=\rho_{1}^{i n}, \\
& \left.\rho^{i n}\right|_{\tau=\tau_{\text {end }}^{\rho^{i n}}}=\rho_{2}^{i n}, \\
& \left.j^{i n}\right|_{\tau=\tau_{\text {start }}^{j^{i n}}}=j_{1}, \\
& \left.j^{i n}\right|_{\tau=\tau_{\text {end }}^{j^{i n}}}=j_{2}, \\
& \begin{array}{l}
\left.\rho^{\text {out }}\right|_{\tau=\tau_{\text {start }}^{\text {out }}}=\rho_{1}^{\text {out }}, \\
\left.\rho^{\text {out }}\right|_{\tau=\tau_{\text {end }}^{\text {out }}}=\rho_{2}^{\text {out }}, \\
\left.j^{\text {out }}\right|_{\tau=\tau_{\text {start }}^{\text {out }}}=j_{1}, \\
\left.j^{\text {out }}\right|_{\tau=\tau_{\text {end }}^{\text {out }}}=j_{2} .
\end{array}
\end{aligned}
$$

Here $\tau_{\text {start/end }}^{\rho^{\text {in } / \text { out }}}$ is the time moment at which variation of mass density at the pipeline's inflow/outflow is start/over, $\tau_{\text {start/end }}^{j^{\text {in }} \text { isut }}$ is the time moment at which variation of flow density at the inflow/outflow is start/over, $\rho_{1 / 2}^{\text {in } / \text { ut }}$ is the value of mass density at the pipeline's inflow/outflow in stationary process $1 / 2, j_{1 / 2}$ is the value of mass flow density in stationary process $1 / 2$.

To satisfy conditions (11), we restrict the class of control functions by monotonic increasing/decreasing ones. Each function $\psi^{\text {in/out }}(\tau), \tau \in\left[0, \tau_{\mathrm{Tr}}\right]$, where $\tau_{\operatorname{Tr}}$ is duration of the transient process, is monotone within the interval $\tau_{\text {var }}^{\psi^{\text {in } / \text { out }}} \equiv\left(\tau_{\text {start }}^{\psi^{\text {in }} / \text { out }}, \tau_{\text {end }}^{\psi^{\text {in }}}{ }^{\text {out }}\right) \subset\left[0, \tau_{\operatorname{Tr}}\right]$ and remains constant values outside $\tau_{\text {var }}^{\psi^{\text {in }} \text { out }}$ :

$$
\begin{aligned}
& d \psi^{\text {in } / \text { out }}(\tau) / d \tau \neq 0, \quad \tau \in \tau_{\mathrm{var}}^{\psi_{\text {in } / \text { out }}}, \\
& \psi^{\text {in/out }}(\tau)=\psi_{1}^{\text {in/out }}=\mathrm{const}, \quad \tau \in\left[0, \tau_{\mathrm{start}}^{\psi^{\text {in } / \text { out }}}\right], \\
& \psi^{\text {in/out }}(\tau)=\psi_{2}^{\text {in } / \text { out }}=\text { const }, \quad \tau \in\left[\tau_{\text {end }}^{\psi^{\text {in }}}, \tau_{\operatorname{Tr}}\right] .
\end{aligned}
$$

where $\psi_{1 / 2}^{\text {in/out }}$ is the value of parameter $\psi$ at the pipeline inflow/outflow in stationary process $1 / 2$.

To satisfy the restrictions we can present the model of control function $\psi(\tau)$ by piece-wise linear approximation

$$
\psi(\tau)=\left\{\begin{array}{l}
\psi_{1}, \quad 0 \leqslant \tau \leqslant \tau_{\text {start }}^{\psi} \\
\psi_{1}+\frac{\psi_{2}-\psi_{1}}{\tau_{\text {end }}^{\psi}-\tau_{\text {start }}^{\psi}}\left(\tau-\tau_{\text {start }}^{\psi}\right), \quad \tau_{\text {start }}^{\psi}<\tau<\tau_{\text {end }}^{\psi}, \\
\psi_{2}, \quad \tau_{\text {end }}^{\psi} \leqslant \tau \leqslant \tau_{\text {Tr }} .
\end{array}\right.
$$

But the first derivative of function (13) has jumps at the moments $\tau=\tau_{\text {start }}^{\psi}$ and $\tau=\tau_{\text {end }}^{\psi}$. To avoid this, we approximate (13) by analytical function of the form

$$
\psi(\tau)=\psi_{1}+\frac{\psi_{2}-\psi_{1}}{2}\left(\tanh \left(a^{\psi}\left(\tau-\tau_{s}^{\psi}\right)\right)+1\right) .
$$

We cannot satisfy the conditions (12) with function (14) exactly. Therefore, instead of conditions $\psi\left(\tau_{\text {start }}^{\psi}\right)=\psi_{1}, \psi\left(\tau_{\text {end }}^{\psi}\right)=\psi_{2}$, we will subordinate functions (14) to the conditions

$$
\left|\psi\left(\tau_{\text {start }}^{\psi}\right)-\psi_{1}\right| \leqslant \varepsilon, \quad\left|\psi_{2}-\psi\left(\tau_{\text {end }}^{\psi}\right)\right| \leqslant \varepsilon,
$$

where $\varepsilon$ is a small positive number. 
This brings to the next formulas for parameters $a^{\psi}$ and $\tau_{s}^{\psi}$ of function (14)

$$
\tau_{s}^{\psi}=\frac{\tau_{\text {start }}^{\psi}+\tau_{\text {end }}^{\psi}}{2}, \quad a^{\psi}=\frac{2}{\tau_{\text {end }}^{\psi}-\tau_{\text {start }}^{\psi}} \operatorname{atanh}\left(1-\frac{2 \varepsilon}{\left|\psi_{2}-\psi_{1}\right|}\right) .
$$

With the use of the models (13) and (14), we can fundamentally constrain the class of control functions $\psi(\tau)$. Each $\psi(\tau) \in\left\{\rho^{\text {in }}(\tau), \rho^{\text {out }}(\tau), j^{\text {in }}(\tau), j^{\text {out }}(\tau)\right\}$ can be defined now by four real constants, namely $-\psi_{1}, \psi_{2}$ and $\tau_{\text {start }}^{\psi}, \tau_{\text {end }}^{\psi}$. The first pair $\psi_{1}, \psi_{2}$ are control parameters of the stationary processes Stationar ${ }_{1}$ and Stationar 2 . The second pair $\tau_{\text {start }}^{\psi}, \tau_{\text {end }}^{\psi}$ are the time parameters, which determine the rate of function $\psi(\tau)$ changing.

Each model $B V P_{K}, K \in\{I, I I, I I I\}$ depends on two controls functions. Hence, the set of control functions of model $\mathrm{K}$ is defined by the set $\Pi_{\mathrm{K}}$ of 8 real parameters:

$$
\begin{aligned}
\Pi_{\mathrm{I}} & =\left\{\rho_{1}^{\text {in }}, \rho_{1}^{\text {out }}, \rho_{2}^{\text {in }}, \rho_{2}^{\text {out }}, \tau_{\text {start }}^{\rho^{\text {in }}}, \tau_{\text {start }}^{\rho^{\text {out }}}, \tau_{\text {end }}^{\rho^{\text {in }}}, \tau_{\text {end }}^{\rho^{\text {out }}}\right\}, \\
\Pi_{\mathrm{II}} & =\left\{\rho_{1}^{\text {in }}, j_{1}, \rho_{2}^{\text {in }}, j_{2}, \tau_{\text {start }}^{\rho^{\text {in }}}, \tau_{\text {start }}^{j^{\text {out }}}, \tau_{\text {end }}^{\rho^{\text {in }}}, \tau_{\text {end }}^{j^{\text {out }}}\right\}, \\
\Pi_{\mathrm{III}} & =\left\{j_{1}, \rho_{1}^{\text {out }}, j_{2}, \rho_{2}^{\text {out }}, \tau_{\text {start }}^{j^{\text {in }}}, \tau_{\text {start }}^{\rho^{\text {out }}}, \tau_{\text {end }}^{j^{\text {in }}}, \tau_{\text {end }}^{\rho^{\text {out }}}\right\} .
\end{aligned}
$$

\section{Internal parameters and functionals of the transient process}

The course of any transient process Transient ${ }_{1-2}$ depends on chosen model $B V P_{K}$ and numerical values of all elements of set $\Pi_{K}$ associated with this model. In the aggregate, $B V P_{K}$ and $\Pi_{K}$ define the algorithm of controlling by the transient process, that can be written as Transient $_{1-2}=$ Transient $_{1-2}\left(\mathrm{BVP}_{\mathrm{K}}, \Pi_{\mathrm{K}}\right)$. It means that assigning different numerical values to the parameters $\tau_{\text {start }}^{\psi}$, $\tau_{\text {end }}^{\psi}$ of a set $\Pi_{\mathrm{K}}$, we define different transient processes, switching-over the pipeline between the same two stationary regimes Stationar ${ }_{1}$ and Stationar ${ }_{2}$ in correspondence with model BVP $\mathrm{P}_{\mathrm{K}}$.

Two internal functions $\rho(\xi, \tau)$ and $j(\xi, \tau)$, being the solution of boundary-value problem $\mathrm{BVP}_{\mathrm{K}}$, fully determine the transient process, controlling by the algorithm $\left\{\mathrm{BVP}_{\mathrm{K}}, \Pi_{\mathrm{K}}\right\}$. The boundary functions used in $\mathrm{BVP}_{\mathrm{K}}$ are external or control functions for this model. For instance, the functions $\rho^{i n}(\tau)$ and $\rho^{\text {out }}(\tau)$, calculated by models (13) or (14) with the use of the data contained in set $\Pi_{\mathrm{I}}$, are control functions for the model BVP . But the functions $j^{\text {in }}(\tau)$ and $j^{\text {out }}(\tau)$ are local internal functions for this model: they can be calculated from the solution $\{\rho(\xi, \tau), j(\xi, \tau)\}$ of the boundary-value problem BVP as $j^{\text {in }}(\tau)=j(0, \tau), j^{\text {out }}(\tau)=j(1, \tau)$.

Similarly, for the model BVP II, $\rho^{\text {in }}(\tau)$ and $j^{\text {out }}(\tau)$ are the control functions: they should be calculated by model (13) or (14) with the use the data containing in the set $\Pi_{\mathrm{II}}$, whereas $j^{\text {in }}(\tau)$ and $\rho^{\text {out }}(\tau)$ are the local internal functions this model. The pair $j^{\text {in }}(\tau)$ and $\rho^{\text {out }}(\tau)$ are the external functions for the model $\mathrm{BVP}_{\mathrm{III}}$, whereas $\rho^{\text {in }}(\tau)$ and $j^{\text {out }}(\tau)$ are the local internal functions for this model.

Besides the local functions of the transient processes, one can consider its integral parameters. We define here some such parameters.

The first one is the total mass $\mathrm{M}(t)$ of the gas that occupies the inner volume of the pipeline at the moment $t$ :

$$
\mathrm{M}(t)=S \int_{0}^{L} D(x, t) d x=\mathrm{M}_{t} \int_{0}^{1} \rho\left(\xi, t / t_{t}\right) d \xi=\mathrm{M}_{t} M\left(t / t_{t}\right),
$$

where $\mathrm{M}_{t}=S L D_{t}, S$ is the area of the pipeline duct's cross-section, $M(\tau)$ is dimensionless mass of the gas:

$$
M(\tau)=\int_{0}^{1} \rho(\xi, \tau) d \xi
$$

Dimensionless mass $M_{1 / 2}$ for process Stationar $_{1 / 2}$ is calculated as

$$
M_{1 / 2}=\int_{0}^{1} \rho_{1 / 2}(\xi) d \xi
$$

Mathematical Modeling and Computing, Vol.6, No. 2, pp. 220-238 (2019) 
The second internal integral parameter we consider here is the power $\mathrm{W}^{R}(t)$ of viscous friction force at the moment $t$ :

$$
\mathrm{W}^{R}(t)=\mathrm{W}_{t}^{R} W^{R}\left(t / t_{t}\right)
$$

where $\mathrm{W}_{t}^{R}=\mathrm{M}_{t} V_{t}^{3} / L, W^{R}(\tau)$ is dimensionless power of viscous friction force:

$$
W^{R}(\tau)=\int_{0}^{1} \beta(\xi) \frac{j^{3}(\xi, \tau)}{\rho^{2}(\xi, \tau)} d \xi
$$

Corresponding parameters for processes Stationar $_{1}$ and Stationar ${ }_{1}$ are calculated as

$$
W_{1 / 2}^{R}=\int_{0}^{1} \beta(\xi) \frac{j_{1 / 2}^{3}(\xi)}{\rho_{1 / 2}^{2}(\xi)} d \xi .
$$

One more internal integral parameter of the transient processes is the work $\mathrm{A}^{R}(t)$ of viscous friction force:

$$
\mathrm{A}^{R}(t)=\int_{0}^{t} \mathrm{~W}^{R}(t) d t=\mathrm{A}_{t}^{R} A^{R}\left(t / t_{t}\right)
$$

where $\mathrm{A}_{t}^{R}=\mathrm{M}_{t} V_{t}^{2}, A^{R}(\tau)$ is dimensionless work of viscous friction force:

$$
A^{R}(\tau) \equiv M a \int_{0}^{\tau} W^{R}(\tau) d \tau=M a \int_{0}^{\tau} \int_{0}^{1} \beta(\xi) \frac{j^{3}(\xi, \tau)}{\rho^{2}(\xi, \tau)} d \xi d \tau .
$$

Now we introduce functional for process Transient ${ }_{1-2}$ i.e. - scalar parameters, the values of which give certain quantitative characteristics of the transient process.

The first is the duration $\tau_{\operatorname{Tr}}$ of the transient process. We define it as

$$
\tau_{\operatorname{Tr}}=\tau_{\mathrm{St}}-\tau_{\text {start }},
$$

where $\tau_{\text {start }}$ is the time moment, when the transient process has been started, $\tau_{\mathrm{St}}$ is a moment of establishing of the stationary process Stationar 2 . Parameter $\tau_{\text {start }}$ can be defined as $\tau_{\text {start }}=\min \left(\tau_{\text {start }}^{\psi^{\text {in }}}, \tau_{\text {start }}^{\psi^{\text {out }}}\right)$.

To detect the moment $\tau_{\text {St }}$ we can use the properties of internal parameters of transient processes. As the process Transient ${ }_{1-2}$ determines the transition from Stationar ${ }_{1}$ to Stationar 2 , its internal parameters are compatible with corresponding parameters of the both stationary processes. In terms of local functions $\rho(\xi, \tau)$ and $j(\xi, \tau)$, this compatibility can by expressed by the relations:

$$
\rho\left(\xi, \tau_{\text {start }}\right)=\rho_{1}(\xi), \quad j\left(\xi, \tau_{\text {start }}\right)=j_{1}, \quad \rho\left(\xi, \tau_{\mathrm{St}}\right)=\rho_{2}(\xi), \quad j\left(\xi, \tau_{\mathrm{St}}\right)=j_{2} .
$$

The first pair of these relations has been used to define the function of the initial conditions. The second one can be applied to determinate the moment $\tau_{\mathrm{St}}$.

If to use the local functions acting on the pipeline ends, then the compatibility relations for models $\mathrm{BVP}_{\mathrm{K}}$ will be

$$
\begin{array}{lllll}
j^{\text {in }}\left(\tau_{\text {start }}\right)=j_{1}, & j^{\text {out }}\left(\tau_{\text {start }}\right)=j_{1}, & j^{\text {in }}\left(\tau_{\mathrm{St}}\right)=j_{2}, & j^{\text {out }}\left(\tau_{\mathrm{St}}\right)=j_{2}, & \mathrm{~K}=\mathrm{I}, \\
j^{\text {in }}\left(\tau_{\text {start }}\right)=j_{1}, & \rho^{\text {out }}\left(\tau_{\text {start }}\right)=\rho_{1}^{\text {out }}, & j^{\text {in }}\left(\tau_{\mathrm{St}}\right)=j_{2}, & \rho^{\text {out }}\left(\tau_{\mathrm{St}}\right)=\rho_{2}^{\text {out }}, & \mathrm{K}=\mathrm{II}, \\
\rho^{\text {in }}\left(\tau_{\text {start }}\right)=\rho_{1}^{\text {in }}, & j^{\text {out }}\left(\tau_{\text {start }}\right)=j_{1}, & \rho^{\text {in }}\left(\tau_{\mathrm{St}}\right)=\rho_{2}^{\text {in }}, & j^{\text {out }}\left(\tau_{\mathrm{St}}\right)=j_{2}, & \mathrm{~K}=\mathrm{III} .
\end{array}
$$

We can use the second pairs of these relations involving parameter $\tau_{\mathrm{St}}$ to determinate it.

It should be stressed that the end phase of the process Transient ${ }_{1-2}$ is of relaxation type. It means that function $j^{i n}(\tau)$, for instance, exponentially approaches to value $j_{2}$ with growing $\tau$ and might never reach it. Hence equations $j^{i n}(\tau)=j_{2}$ will has no solution at finite $\tau$. But at that, function $\left|j^{i n}(\tau)-j_{2}\right|$ will quickly decay with growing $\tau$. Hence we can determine $\tau_{\mathrm{St}}$, for instance, as $\tau_{\mathrm{St}}$ : $\left|j^{\text {in }}(\tau)-j_{2}\right|<\varepsilon$ $\forall \tau \geqslant \tau_{\mathrm{St}}$, where $\varepsilon$ is given small positive real number. In correspondence with that we determine duration $\tau_{\mathrm{St}}$ as 


$$
\begin{array}{lll}
\tau_{\mathrm{St}}=\max \left(\arg \min _{\tau}\left(|| j^{\text {in }}(\tau)-j_{2}|-\varepsilon|\right), \arg \min _{\tau}\left(|| j^{\text {out }}(\tau)-j_{2}|-\varepsilon|\right)\right), & \mathrm{K}=\mathrm{I}, \\
\tau_{\mathrm{St}}=\max \left(\arg \min _{\tau}\left(|| j^{\text {in }}(\tau)-j_{2}|-\varepsilon|\right), \arg \min _{\tau}\left(|| \rho^{\text {out }}(\tau)-\rho_{2}(1)|-\varepsilon|\right)\right), & \mathrm{K}=\mathrm{II}, \\
\tau_{\mathrm{St}}=\max \left(\arg \min _{\tau}\left(|| \rho^{\text {in }}(\tau)-\rho_{2}(0)|-\varepsilon|\right), \arg \min _{\tau}\left(|| j^{\text {out }}(\tau)-j_{2}|-\varepsilon|\right)\right), & \mathrm{K}=\mathrm{III} .
\end{array}
$$

To evaluate the energy expended by the transient process, we will use the work of viscous friction force executed during the process: $\mathrm{A}_{\mathrm{Tr}}^{R}=\mathrm{M}_{t} V_{t}^{2} A_{\mathrm{Tr}}^{R}$, where $A_{\mathrm{Tr}}^{R}$ is dimensionless work defined as

$$
A_{\mathrm{Tr}}^{R}=A^{R}\left(\tau_{\mathrm{St}}\right)-A^{R}\left(\tau_{\text {start }}\right) .
$$

Parameter $A_{\mathrm{Tr}}^{R}$ is a functional of the transient process.

To evaluate the amount of the gas transmitted via the pipeline during the transient process, we will use two parameters. The first one $\mathrm{M}_{\mathrm{Tr}}^{\text {in }}$ defines the mass of gas entered into the pipeline at its inlet and the second $\mathrm{M}_{\mathrm{Tr}}^{\text {out }}$ is the mass of gas delivered into the gas transmission system at the outlet

$$
\mathrm{M}_{\mathrm{Tr}}^{\text {in }}=S \int_{t_{\text {start }}}^{t_{\text {end }}} J^{\text {in }}(t) d t=\mathrm{M}_{t} M_{\mathrm{Tr}}^{\text {in }}, \quad \mathrm{M}_{\mathrm{Tr}}^{\text {out }}=S \int_{t_{\text {start }}}^{t_{\text {end }}} J^{\text {out }}(t) d t=\mathrm{M}_{t} M_{\mathrm{Tr}}^{\text {out }} .
$$

Here $M_{\mathrm{Tr}}^{i n}$ and $M_{\mathrm{Tr}}^{\text {out }}$ are corresponding dimensionless parameters:

$$
M_{\mathrm{Tr}}^{i n}=M a \int_{\tau_{\text {start }}}^{\tau_{\mathrm{St}}} j^{\text {in }}(\tau) d \tau, \quad M_{\mathrm{Tr}}^{\text {out }}=M a \int_{\tau_{\text {start }}}^{\tau_{\mathrm{St}}} j^{\text {out }}(\tau) d \tau
$$

The values, average over the transient process duration, of dimensionless mass flow density at the inlet and outlet

$$
\bar{j}_{\mathrm{Tr}}^{\text {in }}=\frac{M_{\mathrm{Tr}}^{\text {in }}}{M a \cdot \tau_{\mathrm{Tr}}}, \quad \bar{j}_{\mathrm{Tr}}^{\text {out }}=\frac{M_{\mathrm{Tr}}^{\text {out }}}{M a \cdot \tau_{\mathrm{Tr}}}
$$

are also functionals of the transient process. They enable us to compare the efficiency of the transient process and the stationary processes 1 and 2 .

Parameters $\mathrm{Q}_{\mathrm{Tr}}^{\text {in }}$ and $\mathrm{Q}_{\mathrm{Tr}}^{\text {out }}$

$$
\mathrm{Q}_{\mathrm{Tr}}^{i n}=\frac{\mathrm{A}_{T \mathrm{r}}^{R}}{\mathrm{M}_{T \mathrm{r}}^{i n}}=V_{t}^{2} \frac{A_{\mathrm{Tr}}^{R}}{M_{\mathrm{Tr}}^{\text {in }}}=V_{t}^{2} Q_{\mathrm{Tr}}^{\text {in }}, \quad \mathrm{Q}_{\mathrm{Tr}}^{\text {out }}=\frac{\mathrm{A}_{T \mathrm{r}}^{R}}{\mathrm{M}_{T \mathrm{r}}^{\text {out }}}=V_{t}^{2} \frac{A_{\mathrm{Tr}}^{R}}{M_{\mathrm{Tr}}^{\text {out }}}=V_{t}^{2} Q_{\mathrm{Tr}}^{\text {out }}
$$

define the specific energy, expended for entering the gas into the pipeline through the inlet and delivered it into the gas transmission system through the outlet of the pipeline.

Dimensionless parameters $Q_{\mathrm{Tr}}^{\text {in }}$ and $Q_{\mathrm{Tr}}^{\text {out }}$ are functionals of the transient process. They can be used to evaluate the power efficiency of the transient process $\operatorname{Transient}_{1-2}\left(\mathrm{BVP}_{\mathrm{K}}, \Pi_{\mathrm{K}}\right)$ by comparing them to corresponding parameters $Q_{1}$ and $Q_{2}$ of the stationary processes Stationar ${ }_{1}$ and Stationar calculated as

$$
Q_{1}=\frac{W_{1}}{j_{1}}=j_{1}^{2} \int_{0}^{1} \frac{\beta(\xi)}{\rho_{1}^{2}(\xi)} d \xi, \quad Q_{2}=\frac{W_{2}}{j_{2}}=j_{2}^{2} \int_{0}^{1} \frac{\beta(\xi)}{\rho_{2}^{2}(\xi)} d \xi
$$

\section{Case study of the models and algorithms for control by transient processes}

In this section, we consider the results of the numerical study of transient processes executed due to three models $\mathrm{BVP}_{\mathrm{K}}$ by various algorithms. Totally, we have studied 12 various algorithms. In all cases, the same two stationary processes, called "low flow stationary process" and "high flow stationary process", were used. They are determined by the next control parameters:

- low flow stationary process: $\rho_{1}^{\text {in }}=0.7, \rho_{1}^{\text {out }}=0.3, j_{1}=0.6372$;

- high flow stationary process: $\rho_{2}^{\text {in }}=0.9, \rho_{2}^{\text {out }}=0.3, j_{2}=0.8443$. 
In Fig. 1 one can see the dimensionless mass density and flow density in the pipeline for these stationary processes.

In all cases, we used the model for control functions in form (14).

The algorithms switching-over the pipeline from the low flow stationary process into the high flow stationary process as well as that switching-over the pipeline in reverse direction were considered.

The problems $\mathrm{BVP}_{\mathrm{K}}, \mathrm{K}=\mathrm{I}$, II, III were solved numerically. For that, a uniform grid $X$ was created on section $[0,1]$. Then, with the use of finite difference method, the problems $\mathrm{BVP}_{\mathrm{K}}$ were reduced to corresponding initial value problems $I V P_{K}$ for systems of ordinary differential equations relative to the node values $\rho\left(\xi_{i}, \tau\right) j\left(\xi_{i}, \tau\right), \xi_{i} \in X$ of the key functions. The problems IVP $_{\mathrm{K}}$ were solved by the Runge-Kutta method

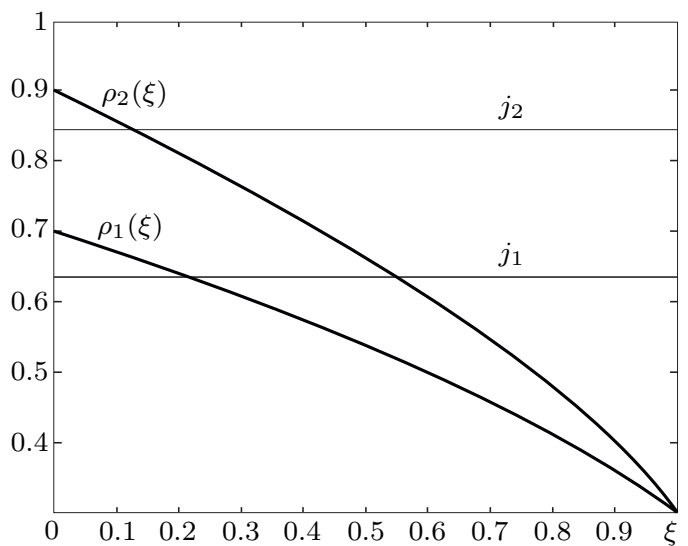

Fig. 1. The graphs of control parameters for two stationary processes used in the case studies. applying the known algorithm RKF-45 [11]. Obtained in such manner solutions contain node values $\rho\left(\xi_{i}, \tau_{j}\right)$ and $j\left(\xi_{i}, \tau_{j}\right)$ of the key functions at discrete time moments $\tau_{j}$. The solutions were used to calculate the internal parameters of transient processes.

The results of numerical studies presented in this chapter were obtained for the horizontal pipeline with length $100 \mathrm{~km}$ and diameter $1.420 \mathrm{~m}$. The next values for the typical gas state parameters were used: pressure $P_{t}=6.665 \mathrm{MPa}$, temperature $T_{t}=300 \mathrm{~K}$, density $D_{t}=48.2016 \mathrm{~kg} / \mathrm{m}^{3}$. The typical mass flow density was taken $J_{t}=467.8117 \mathrm{~kg} /\left(\mathrm{m}^{2} \cdot \mathrm{s}\right)$, typical gas velocity $V_{t}=9.7053 \mathrm{~m} / \mathrm{s}$, typical sonic velocity, Mach number and time period are correspondingly: $C_{t}=396.6253 \mathrm{~m} / \mathrm{s}, M a=0.0245$ and $t_{t}=252.1271 \mathrm{~s}$. The equivalent height of inner surface irregularities of the pipeline wall was taken equal to $3.3 \cdot 10^{-5}$.

\subsection{Control model BVP,}

External functions $\rho^{\text {in }}(\tau)$ and $\rho^{\text {out }}(\tau)$ are control in model BVP. First, we considered the case of pipeline transition from the state with low flow into that with high flow. Since outlet density of both stationary processes Stationar ${ }_{1}$ and Stationar 2 are the same, so $\rho^{\text {out }}(\tau)=$ const. Therefore, it was enough to define the values of time control parameters in set $\Pi_{I}$ only for function $\rho^{\text {in }}(\tau)$. So, we chose this set in the form:

$$
\Pi_{\mathrm{I}}^{1}=\left\{\rho_{1}^{\text {in }}=0.7, \rho_{1}^{\text {out }}=0.3, \rho_{2}^{\text {in }}=0.9, \rho_{1}^{\text {out }}=0.3, \tau_{\text {start }}^{\rho^{\text {in }}}=25, \tau_{\text {end }}^{\rho^{\text {in }}}=75\right\} .
$$

In Fig. 2 , the graphs of functions of the transient process Transient $_{1-2}\left(\mathrm{BVP}_{\mathrm{I}}, \Pi_{\mathrm{I}}^{1}\right)$ are shown. In Fig. $2 a$ one can see the graphs of the control function $\rho^{i n}(\tau)$ and two end internal functions $-j^{i n}(\tau)$ and $j^{\text {out }}(\tau)$, calculated with the use of obtained solution. The duration of the transient process Transient $_{1-2}\left(\mathrm{BVP}_{\mathrm{I}}, \Pi_{\mathrm{I}}^{1}\right)$, calculated due to the first formula (28) with precision $\varepsilon=0.02$ equals 68.18.

Fig. $2 b$ illustrates how integral parameters - dimensionless mass $M$ of the gas filling the pipeline (curve 1) and dimensionless power of viscous friction force $W^{R}$ (curve 2) change with time $\tau$.

The graphs in Figs. $2 c$ and $2 d$ show distributions of dimensionless mass density and flow in the pipeline at various time moments.

To estimate impact the rate of control parameter $\rho^{\text {in }}$ changing on the transient process, we applied control function $\rho^{i n}(\tau)$ with shorter period $\Delta \tau^{\rho^{i n}} \equiv \tau_{\text {end }}^{\rho^{i n}}-\tau_{\text {start }}^{\rho^{i n}}$ of variation - instead of 50 we chose $\Delta \tau^{\rho^{i n}}=30$, other control parameters have remained the same:

$$
\Pi_{\mathrm{I}}^{2}=\left\{\rho_{1}^{\text {in }}=0.7, \rho_{1}^{\text {out }}=0.3, \rho_{2}^{\text {in }}=0.9, \rho_{1}^{\text {out }}=0.3, \tau_{\text {start }}^{\rho^{\text {in }}}=25, \tau_{\text {end }}^{\rho^{\text {in }}}=55\right\} .
$$

In Fig. 3 we can see the plots of the functions of the transient process Transient $t_{1-2}\left(B \mathrm{P}_{\mathrm{I}}, \Pi_{\mathrm{I}}^{2}\right)$. This process duration equals 53.84 . 

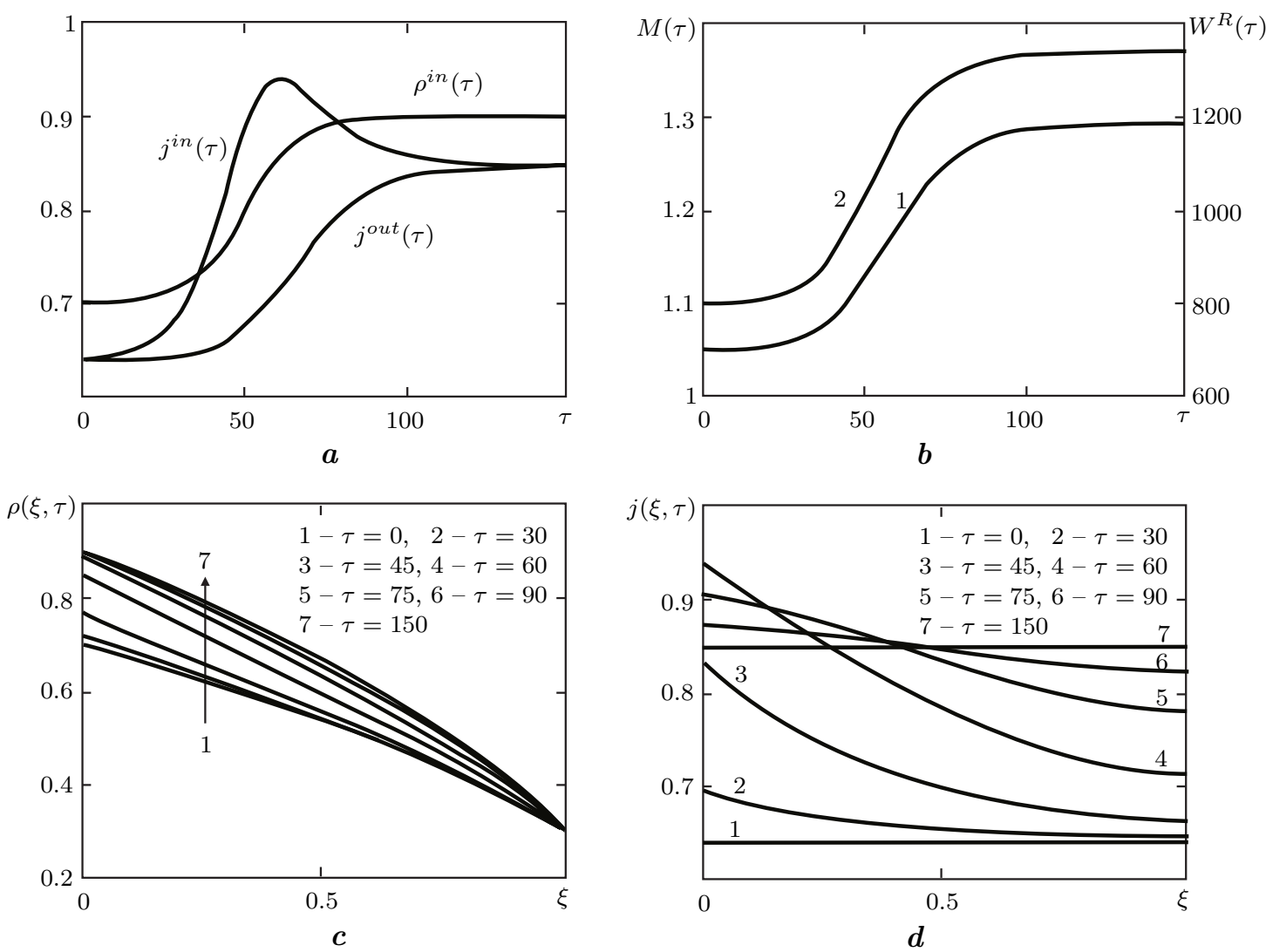

Fig. 2. The graphs of the functions of the transient process $\operatorname{Transient}_{1-2}\left(B \mathrm{BP}_{\mathrm{I}}, \Pi_{\mathrm{I}}^{1}\right)$.
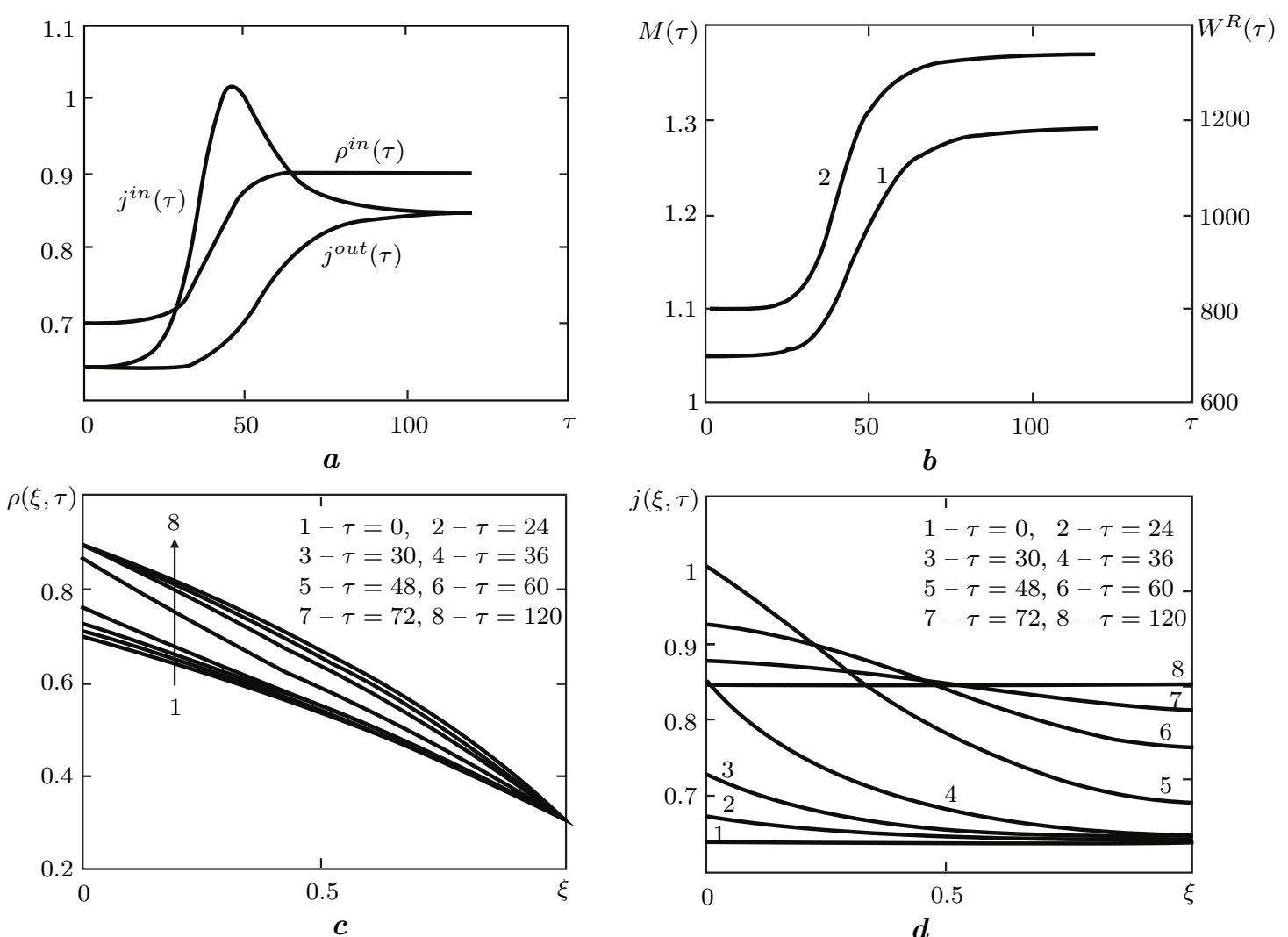

Fig. 3. The graphs of the functions of the transient process $\operatorname{Transient}_{1-2}\left(\mathrm{BVP}_{\mathrm{I}}, \Pi_{\mathrm{I}}^{2}\right)$. 

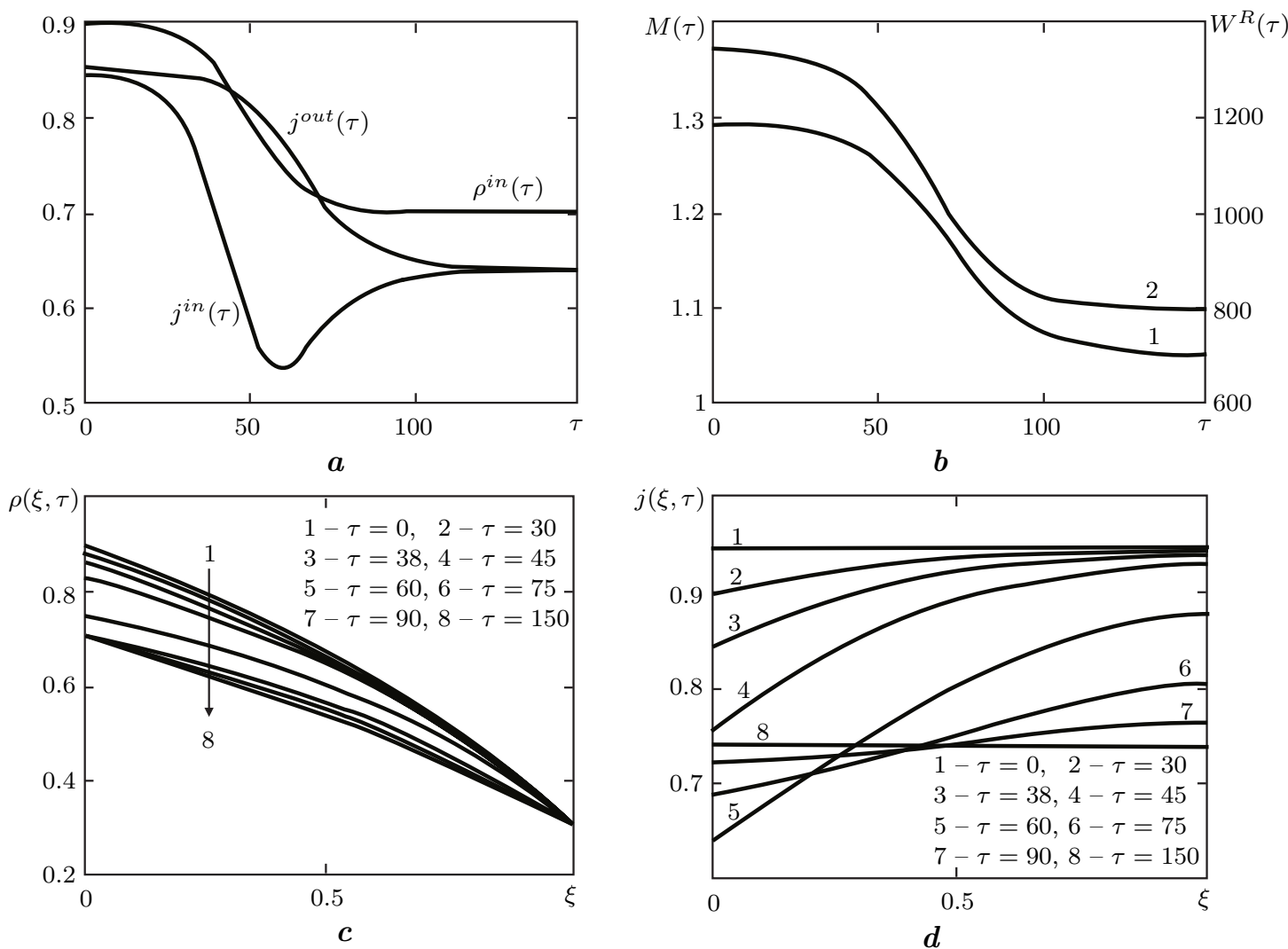

Fig. 4. The graphs of functions of the transient process Transient $_{2-1}\left(B V P_{I}, \Pi_{I}^{3}\right)$.
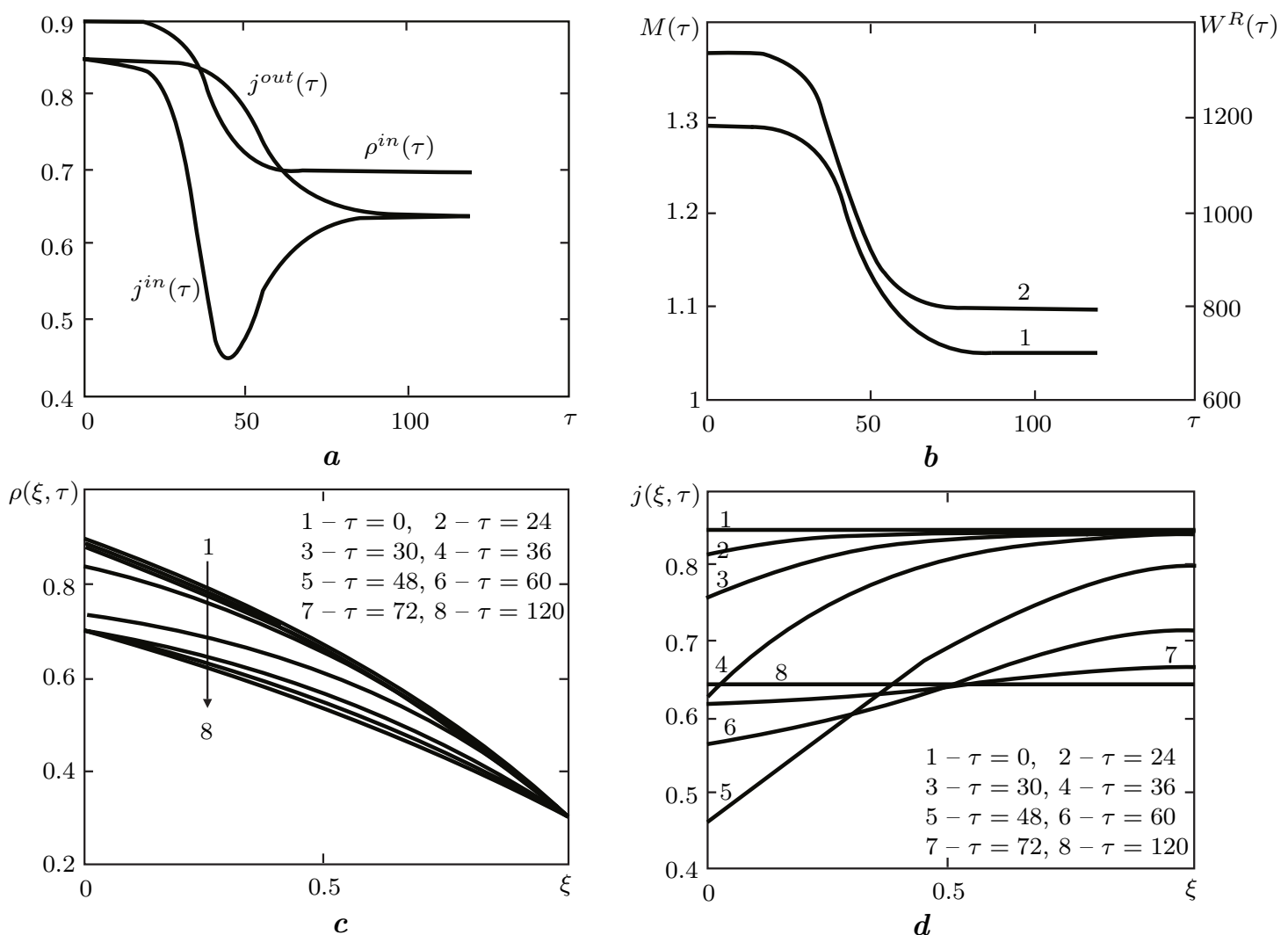

Fig. 5. The graphs of functions of the transient process $\operatorname{Transient}_{2-1}\left(B V P_{I}, \Pi_{I}^{4}\right)$. 
We also studied two control algorithms for switching-over the pipeline from the stationary state of high flow into that of low flow. In the first one, the period $\Delta \tau^{\rho^{i n}}$ of control function varying was taken 50 , in another - 30:

$$
\begin{aligned}
& \Pi_{\mathrm{I}}^{3}=\left\{\rho_{1}^{\text {in }}=0.9, \rho_{1}^{\text {out }}=0.3, \rho_{2}^{\text {in }}=0.7, \rho_{1}^{\text {out }}=0.3, \tau_{\text {start }}^{\rho^{\text {in }}}=25, \tau_{\text {end }}^{\rho^{\text {in }}}=75\right\} \\
& \Pi_{\mathrm{I}}^{4}=\left\{\rho_{1}^{\text {in }}=0.9, \rho_{1}^{\text {out }}=0.3, \rho_{2}^{\text {in }}=0.7, \rho_{1}^{\text {out }}=0.3, \tau_{\text {start }}^{\rho^{\text {in }}}=25, \tau_{\text {end }}^{\rho^{\text {in }}}=55\right\}
\end{aligned}
$$

The durations of these process are 66.22 and 49.93 correspondingly. The graphs of the transient processes Transient $_{2-1}\left(\mathrm{BVP}_{\mathrm{I}}, \Pi_{\mathrm{I}}^{3}\right)$ and Transient $_{2-1}\left(\mathrm{BVP}_{\mathrm{I}}, \Pi_{\mathrm{I}}^{3}\right)$ are shown on Figs. 4 and 5 correspondingly.

\subsection{Control model BVP III}

External functions $j^{\text {in }}(\tau)$ and $\rho^{\text {out }}(\tau)$ are the control in this model. Outlet density of both stationary processes are the same, so it was enough to define the values of time control parameters in sets $\Pi_{\text {III }}$ only for function $j^{i n}(\tau)$. We considered here two cases with control parameters:

$$
\begin{aligned}
& \Pi_{\mathrm{III}}^{1}=\left\{j_{1}^{\text {in }}=0.6372, \rho_{1}^{\text {out }}=0.3, j_{2}^{\text {in }}=0.8443, \rho_{2}^{\text {out }}=0.3, \tau_{\text {start }}^{j^{\text {in }}}=25, \tau_{\text {end }}^{j^{\text {in }}}=75\right\}, \\
& \Pi_{\mathrm{III}}^{2}=\left\{j_{1}=0.8443, \rho_{1}^{\text {out }}=0.3, j_{2}=0.6372, \rho_{2}^{\text {out }}=0.3, \tau_{\text {start }}^{j^{\text {in }}}=25, \tau_{\text {end }}^{j^{\text {in }}}=75\right\} .
\end{aligned}
$$

The graphs of these transient processes are shown on Figs. 6 and 7 correspondingly.
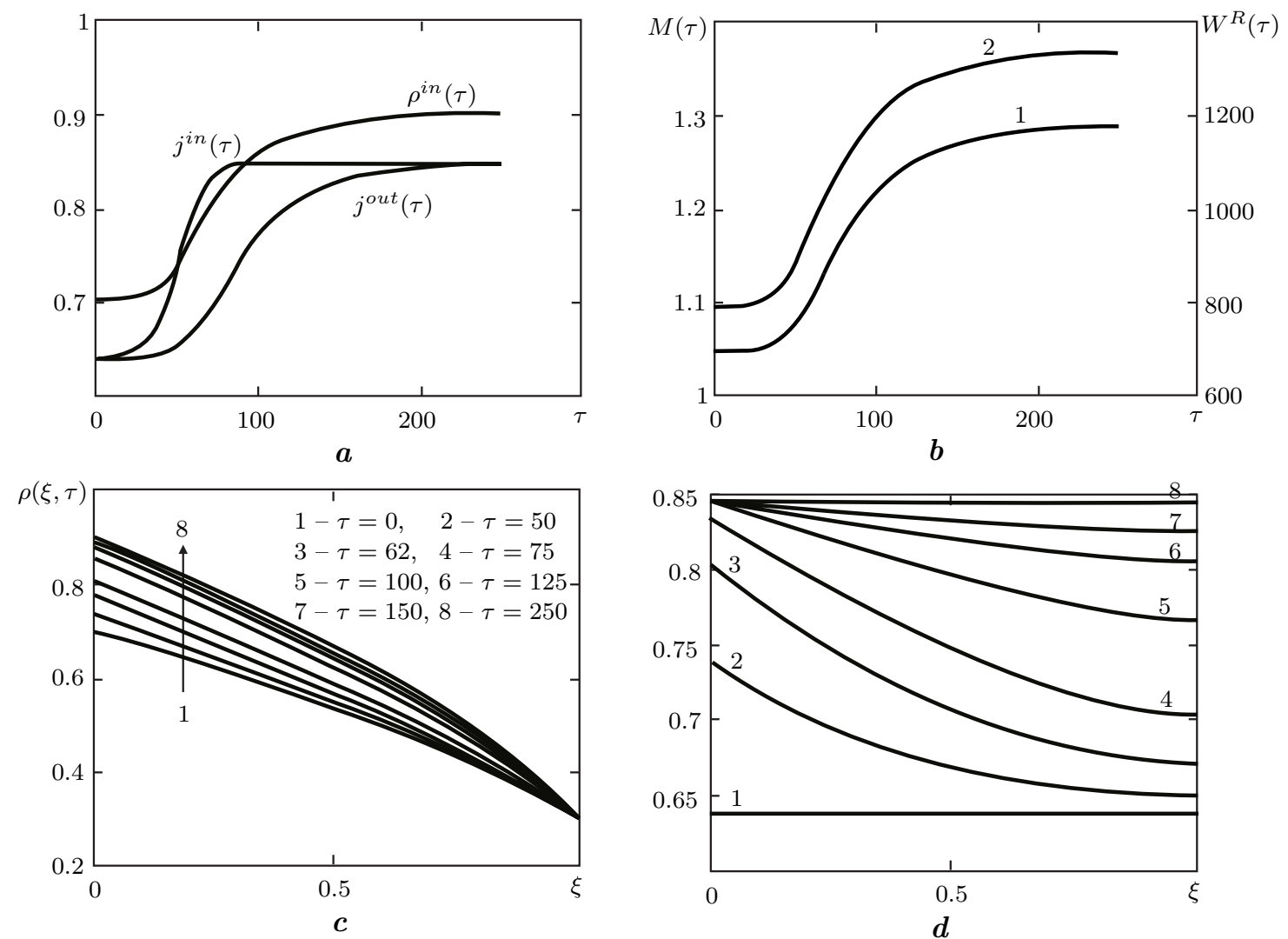

Fig. 6. The graphs of functions of the transient process Transient $_{2-1}\left(B V P_{I I I}, \Pi_{I I I}^{1}\right)$.

The durations of these transient processes are 125.75 and 123.50 correspondingly.

Mathematical Modeling and Computing, Vol. 6, No. 2, pp. 220-238 (2019) 

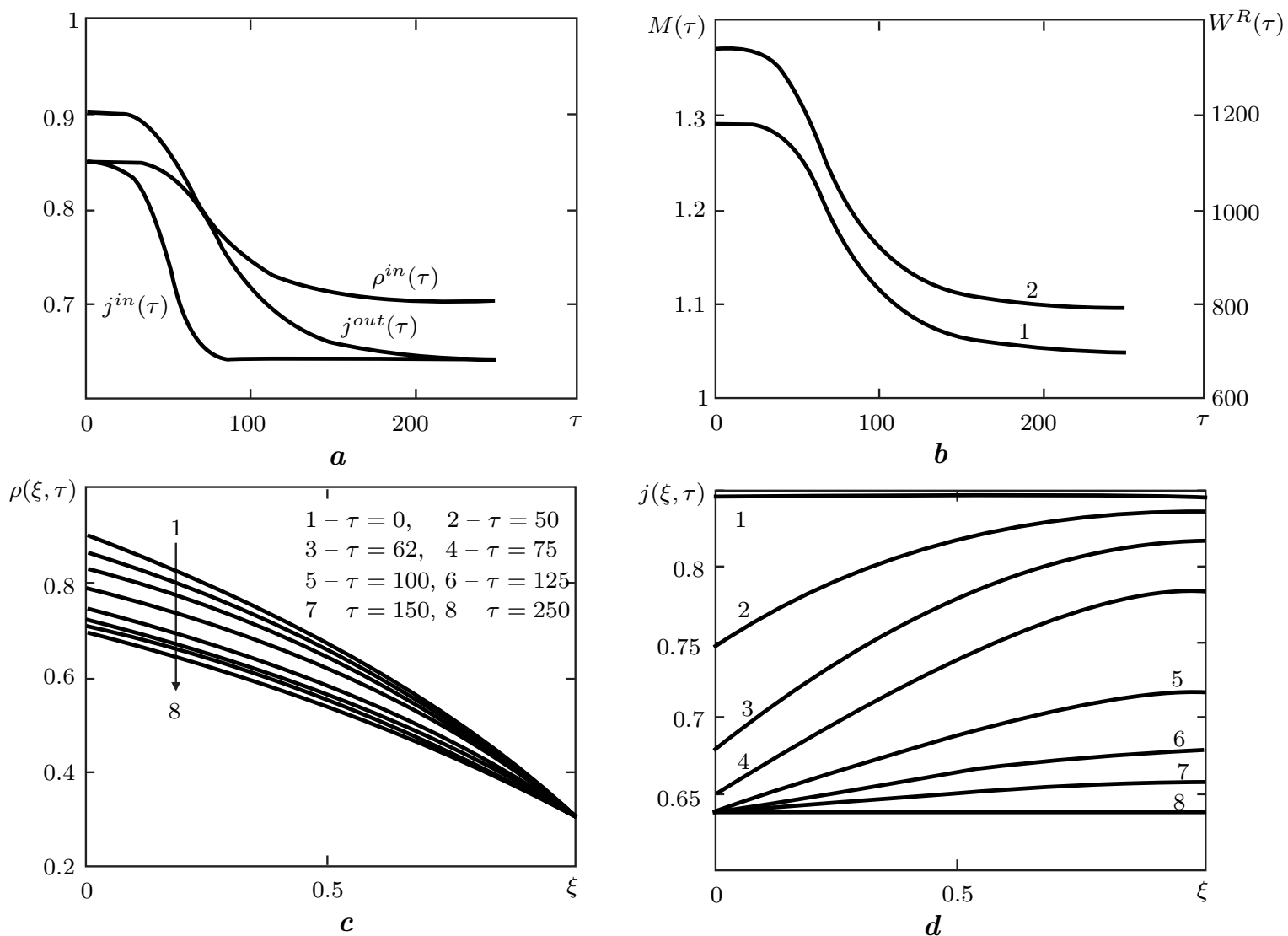

Fig. 7. The graphs of functions of the transient process $\operatorname{Transient}_{2-1}\left(\mathrm{BVP}_{\mathrm{III}}, \Pi_{\mathrm{I}}^{2}\right)$.

\subsection{Control model BVP॥I}

In this model external functions $\rho^{\text {in }}(\tau)$ and $j^{\text {out }}(\tau)$ control by transients processes.

First, we considered the case, when both control functions start at the same time moment and durations of their variation are equal: $\tau_{\text {delay }}^{\rho^{\text {in }}}=\tau_{\text {delay }}^{j^{\text {out }}}, \Delta \tau^{\rho^{\text {in }}}=\Delta \tau^{j^{\text {out }}}$. For that, we chose the next set of control parameter for external functions:

$$
\Pi_{\mathrm{II}}^{1}=\left\{\rho_{1}^{\text {in }}=0.7, j_{1}=0.6372, \rho_{2}^{\text {in }}=0.9, j_{2}=0.8443, \tau_{\text {start }}^{\rho^{\text {in }}}=25, \tau_{\text {end }}^{\rho^{\text {in }}}=75, \tau_{\text {start }}^{j^{\text {out }}}=25, \tau_{\text {end }}^{j^{\text {out }}}=75\right\} .
$$

The graphs of this transient process functions are shown on Fig. 8.

On Fig. $8 a$ we can see the graphs of two control functions $-\rho^{i n}(\tau)$ and $j^{\text {out }}(\tau)$, and two end internal functions of the process $-j^{i n}(\tau)$ and $\rho^{\text {out }}(\tau)$. The duration of the process is $\tau_{\operatorname{Tr}} \approx 153.70$.

In Fig. 8b, where internal integral functions $M(\tau)$ and $W^{R}(\tau)$ are presented, we observe the essential raising of the power of viscous friction force at the beginning phase of the process Transient $_{1-2}\left(B V P_{I I}, \Pi_{\mathrm{II}}^{1}\right)$. We can explain this by the rapid raising of the flow at the pipeline's inflow in this phase (see Fig. 8d) and the corresponding growth of the friction force.

To estimate the impact of the control function $j^{\text {out }}(\tau)$ rate on the transient process, we shortened the interval $\Delta \tau^{\text {out }}$ of its varying from 50 till 30, taking the next set of parameters for the control functions

$$
\Pi_{\mathrm{II}}^{2}=\left\{\rho_{1}^{\text {in }}=0.7, j_{1}=0.6372, \rho_{2}^{\text {in }}=0.9, j_{2}=0.8443, \tau_{\text {start }}^{\rho^{\text {in }}}=25, \tau_{\text {end }}^{\rho^{\text {in }}}=75, \tau_{\text {start }}^{j^{\text {out }}}=25, \tau_{\text {end }}^{j^{\text {out }}}=55\right\} .
$$

The graphs of this transient process are shown on Fig. 9. 

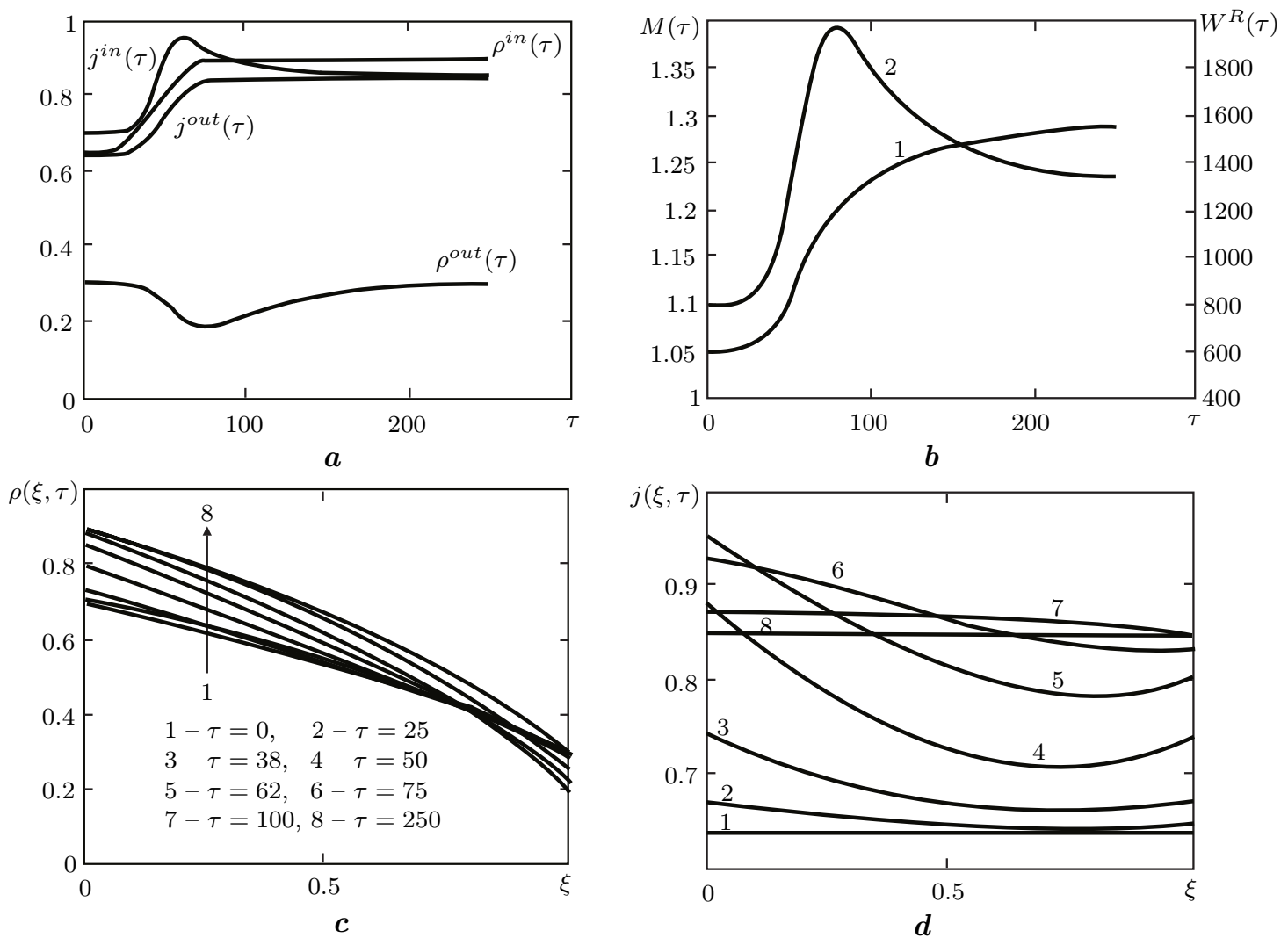

Fig. 8. The graphs of functions of the transient process Transient $t_{1-2}\left(B V P_{I I}, \Pi_{I I}^{1}\right)$.
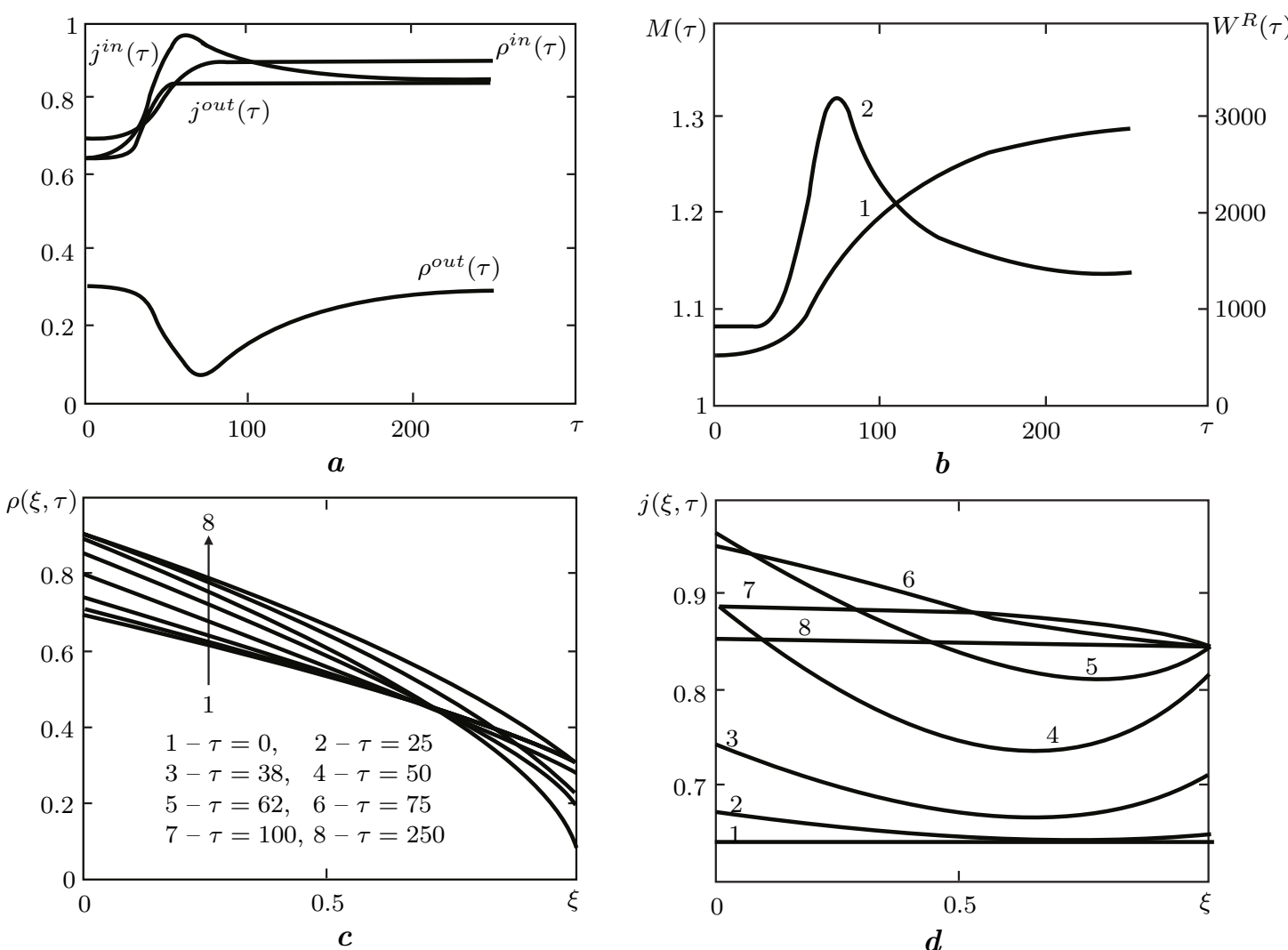

Fig. 9. The graphs of functions of the transient process $\operatorname{Transient}_{1-2}\left(B \vee P_{I I}, \Pi_{I I}^{2}\right)$. 
The duration of the process is $\tau_{\mathrm{Tr}} \approx 175.67$.

Here we can observe intense decreasing of outflow density (see Fig. $9 a$ ) and more rapid growth of the power of viscous friction force at the beginning phase of the transient process (see Fig. 9b).

The next numeric experiment was intended to study the influence of time shift between control functions acting at the pipeline's inflow and outflow on the transient process. For that, we considered an algorithm of switching-over of the pipeline from the state of low flow into that of high flow in the case when the control function at outflow is lag in relation to that at the inflow. The next set of parameters of control functions was used in this case

$$
\Pi_{\mathrm{II}}^{3}=\left\{\rho_{1}^{\text {in }}=0.7, j_{1}=0.6372, \rho_{2}^{\text {in }}=0.9, j_{2}=0.8443, \tau_{\text {start }}^{\rho^{\text {in }}}=25, \tau_{\text {end }}^{\rho^{\text {in }}}=75, \tau_{\text {start }}^{j^{\text {out }}}=50, \tau_{\text {end }}^{j^{\text {out }}}=80\right\} .
$$

The graphs of the transient process functions are shown in Fig. 10.
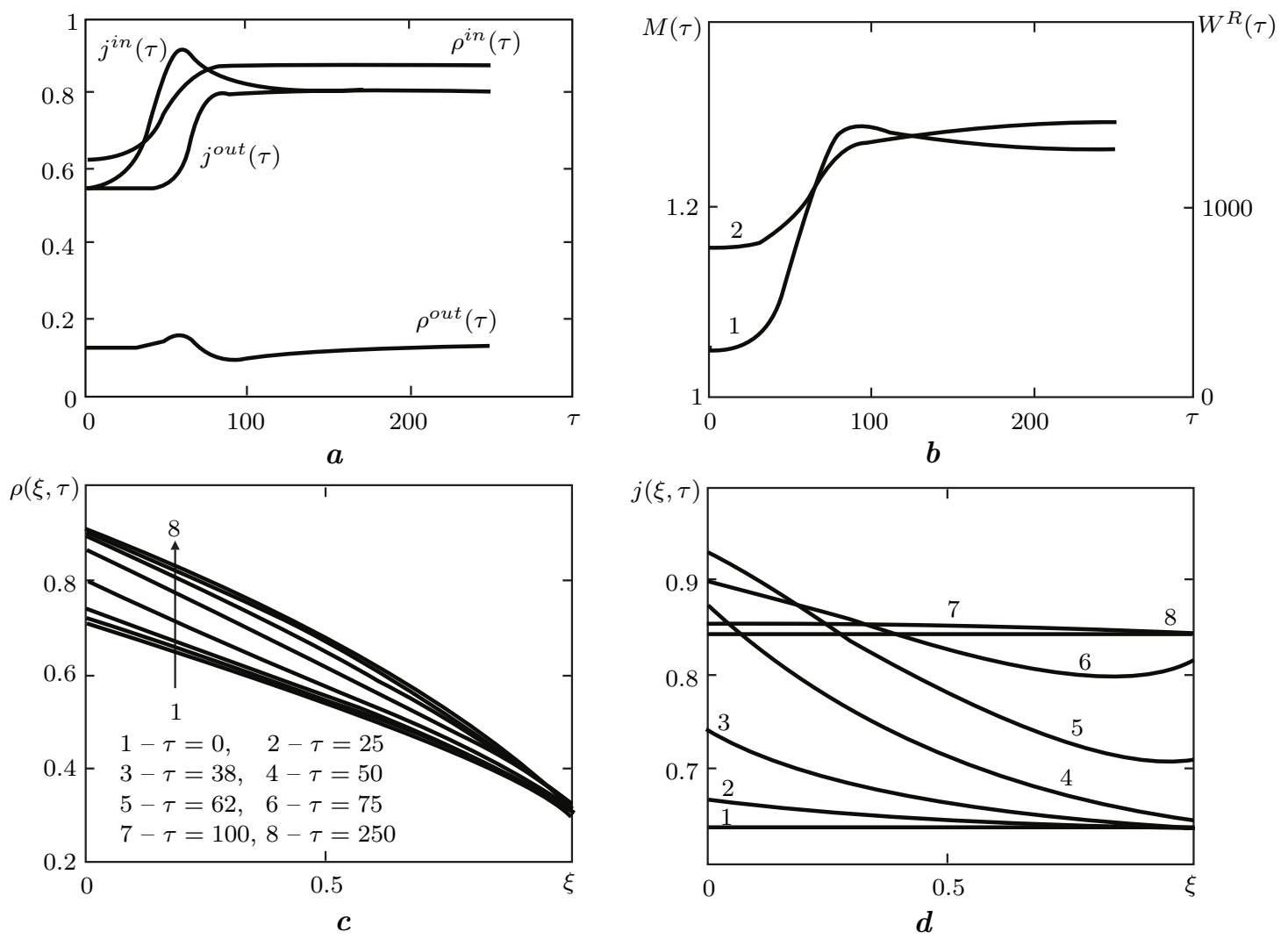

Fig. 10. The graphs of functions of the transient process Transient $t_{1-2}\left(\mathrm{BVP}_{\mathrm{II}}, \Pi_{\mathrm{II}}^{3}\right)$.

As we can see from the graphs, the lag in 25 units of dimensionless time between control functions, acting at the inflow and outflow of the pipeline, makes the transient process more "quiet". That appears, in particular, in more smooth dependencies $\rho^{\text {out }}(\tau)$ and $W^{R}(\tau)$ as compared to those arising in the process Transient $_{1-2}\left(\mathrm{BVP}_{\mathrm{II}}, \Pi_{\mathrm{II}}^{2}\right)$.

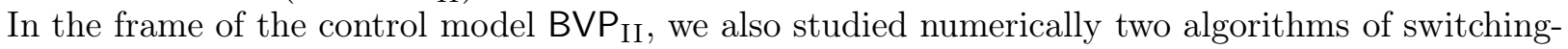
over the pipeline from the state of high flow into the state of low flow.

First, we considered the case of synchronous starts of control functions acting at the inflow and outflow, using the next set of control parameters:

$$
\Pi_{\mathrm{II}}^{4}=\left\{\rho_{1}^{\text {in }}=0.9, j_{1}=0.8443, \rho_{2}^{\text {in }}=0.7, j_{2}=0.6372, \tau_{\text {start }}^{\rho^{\text {in }}}=25, \tau_{\text {end }}^{\rho^{\text {in }}}=75, \tau_{\text {start }}^{j^{\text {out }}}=25, \tau_{\text {end }}^{j^{\text {out }}}=55\right\} .
$$

The graphs of the functions of the transient process are shown in Fig. 11. 

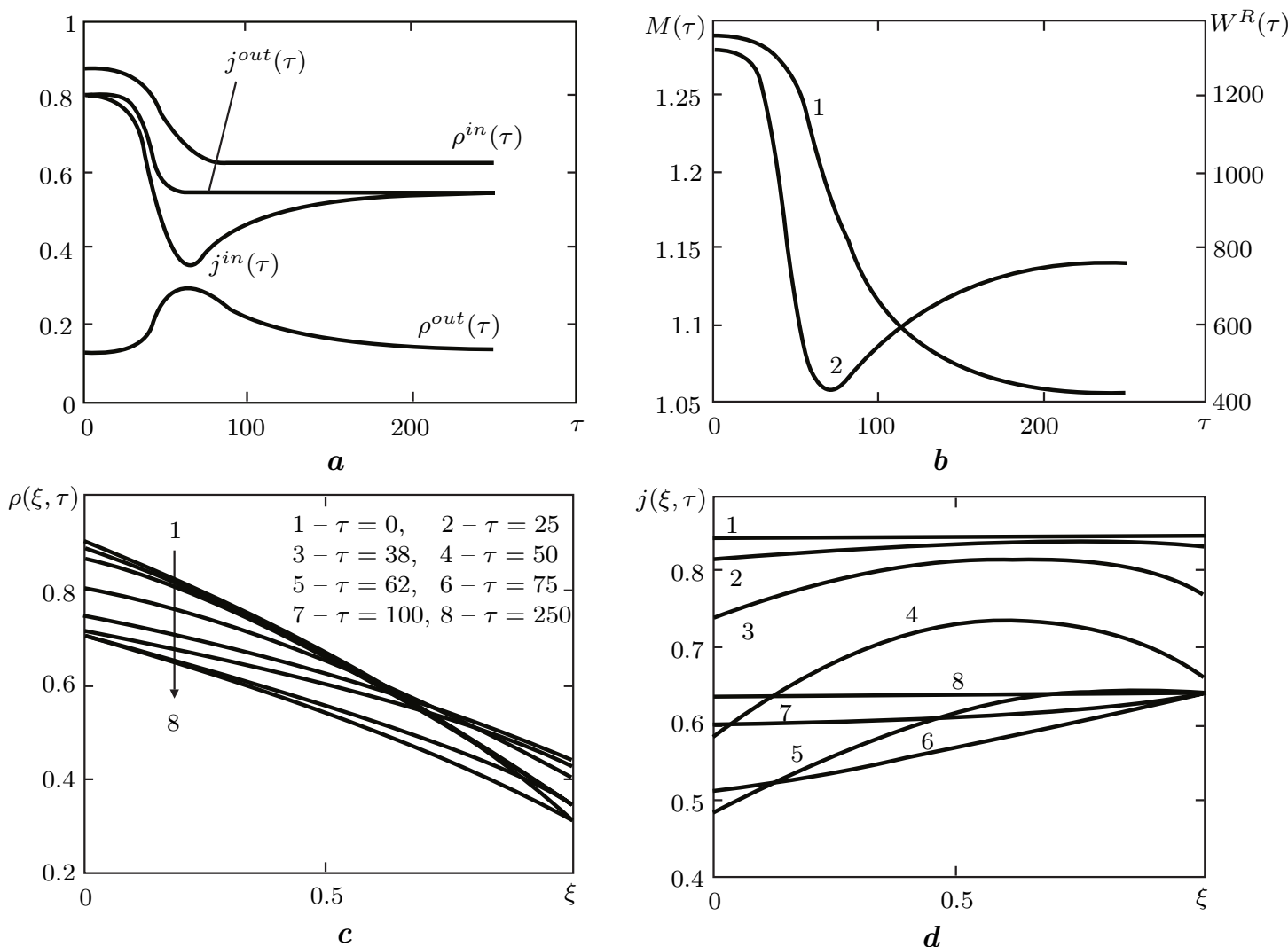

Fig. 11. The graphs of functions of the transient process $\operatorname{Transient}_{1-2}\left(\mathrm{BVP}_{\mathrm{II}}, \Pi_{\mathrm{II}}^{4}\right)$.
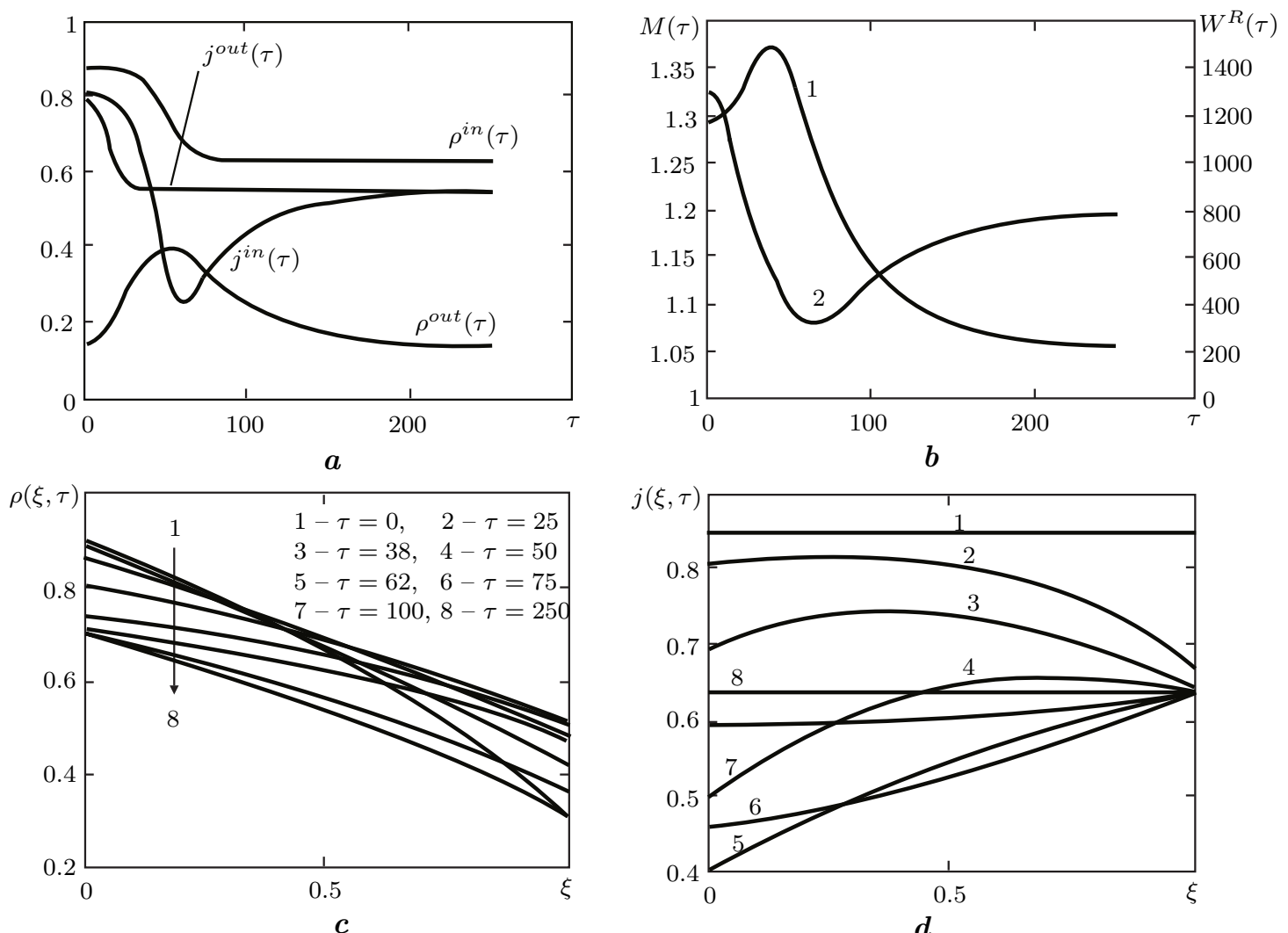

Fig. 12. The graphs of functions of the transient process Transient $t_{1-2}\left(B V P_{I I}, \Pi_{I I}^{5}\right)$. 
The duration of this transient process is $\tau_{\mathrm{Tr}} \approx 169.56$.

The next case was intended to estimate how lag in the start of control function acting at the pipeline inflow will impact the transient process. We used the next set of control parameters for that

$$
\Pi_{\mathrm{II}}^{5}=\left\{\rho_{1}^{\text {in }}=0.9, j_{1}=0.8443, \rho_{2}^{\text {in }}=0.7, j_{2}=0.6372, \tau_{\text {start }}^{\rho^{\text {in }}}=25, \tau_{\text {end }}^{\rho^{\text {in }}}=75, \tau_{\text {start }}^{j^{\text {out }}}=0, \tau_{\text {end }}^{j^{\text {out }}}=30\right\} .
$$

The graphs of the functions of the transient process are shown in Fig. 12.

The duration of this process is $\tau_{\operatorname{Tr}} \approx 169.56$.

One more case we considered was intended to estimate how the lag of the start of the control function, acting at the pipeline's outlet, impacts the transient process. The next set of control parameters was used for that

$$
\Pi_{\mathrm{II}}^{6}=\left\{\rho_{1}^{\text {in }}=0.9, j_{1}=0.8443, \rho_{2}^{\text {in }}=0.7, j_{2}=0.6372, \tau_{\text {start }}^{\rho^{\text {in }}}=25, \tau_{\text {end }}^{\rho^{\text {in }}}=75, \tau_{\text {start }}^{j^{\text {out }}}=50, \tau_{\text {end }}^{j^{\text {out }}}=80\right\} .
$$

By this algorithm, the control function $j^{\text {out }}(\tau)$, acting at the outflow, starts with a lag of 25 units of dimensionless time in relation to control function $\rho^{i n}(\tau)$ at the inflow.

The graphs of the functions of the transient process are shown in Fig. 13.
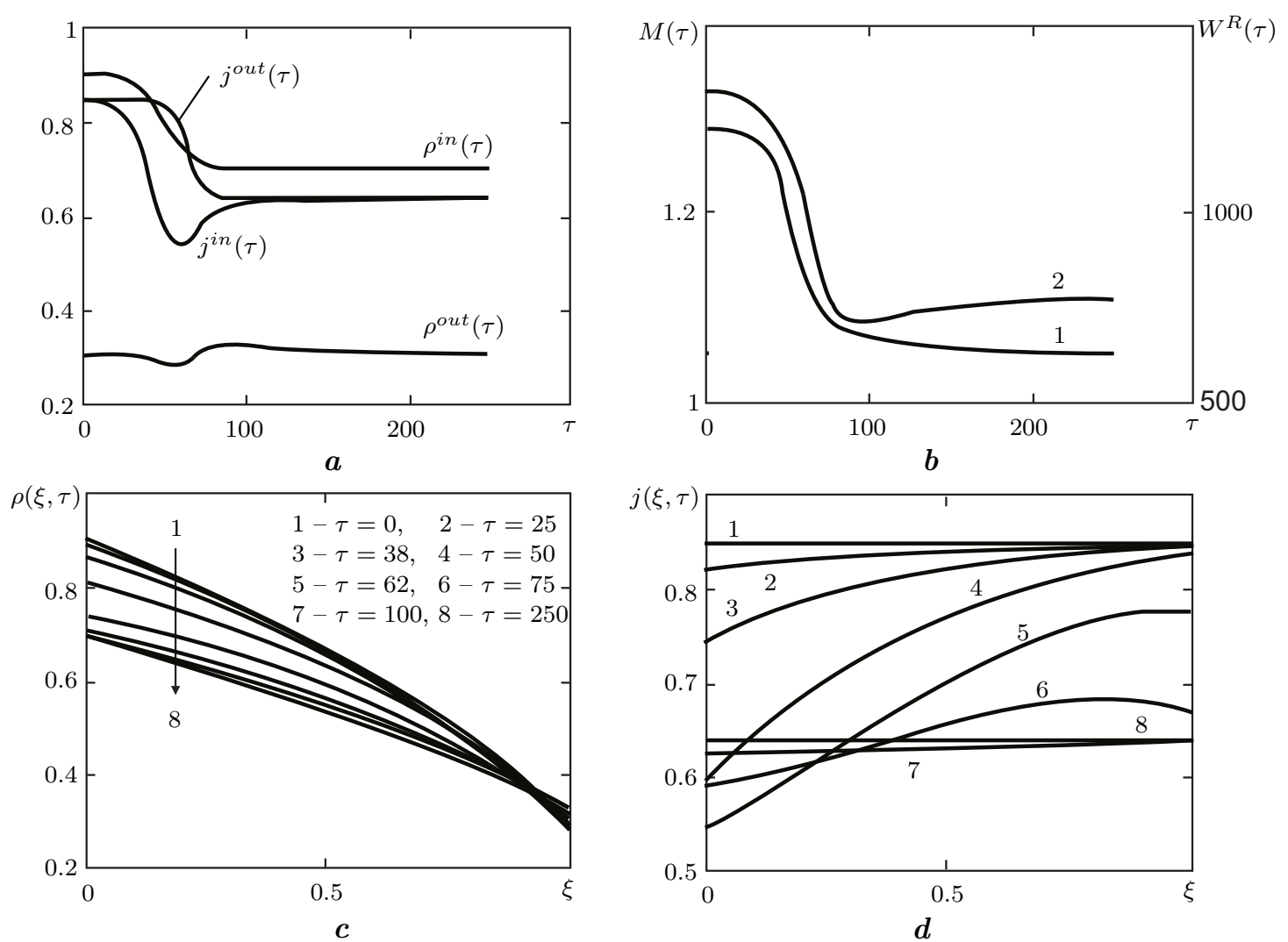

Fig. 13. The graphs of functions of the transient process Transient ${ }_{1-2}\left(B V P_{I I}, \Pi_{I I}^{6}\right)$.

Comparing the graphs in Fig. 13 with corresponding that presented in Fig. 12, we can conclude, that the delay in control at outflow makes the transient process more "quiet" and cuts its duration.

\subsection{Quantitative comparison of the transient processes}

To compare considered in this chapter algorithms, we calculated for each of them the functionals, introduced by formulas (28), (31), (32), (34). In Table 1 the control parameters of the transient processes are presented. In Table 2 the values of functionals for these processes are consolidated. 
Table 1. The control parameters of the transient processes.

\begin{tabular}{|c|c|c|c|c|}
\hline Algorithm & Direction & $\Delta \tau^{\text {in }}$ & $\Delta \tau^{\text {out }}$ & $\tau_{\text {delay }}^{\text {out }}$ \\
\hline BVP $_{\mathrm{I}}, \Pi_{\mathrm{I}}^{1}$ & $1-2$ & 50 & - & 0 \\
\hline $\mathrm{BVP}_{\mathrm{I}}, \Pi_{\mathrm{I}}^{2}$ & $1-2$ & 30 & - & 0 \\
\hline $\mathrm{BVP}_{\mathrm{I}}, \Pi_{\mathrm{I}}^{3}$ & $2-1$ & 50 & - & 0 \\
\hline $\mathrm{BVP}_{\mathrm{I}}, \Pi_{\mathrm{I}}^{4}$ & $2-1$ & 30 & - & 0 \\
\hline $\mathrm{BVP}_{\mathrm{II}}, \Pi_{\mathrm{II}}^{1}$ & $1-2$ & 50 & - & 0 \\
\hline $\mathrm{BVP}_{\mathrm{III}}, \Pi_{\mathrm{II}}^{2}$ & $2-1$ & 50 & - & 0 \\
\hline $\mathrm{BVP}_{\mathrm{II}}, \Pi_{\mathrm{II}}^{1}$ & $1-2$ & 50 & 50 & 0 \\
\hline $\mathrm{BVP}_{\mathrm{II}}, \Pi_{\mathrm{II}}^{2}$ & $1-2$ & 50 & 30 & 0 \\
\hline BVP $_{\mathrm{II}}, \Pi_{\mathrm{II}}^{3}$ & $1-2$ & 50 & 30 & 25 \\
\hline $\mathrm{BVP}_{\mathrm{II}}, \Pi_{\mathrm{II}}^{4}$ & $2-1$ & 50 & 30 & 0 \\
\hline BVP $_{\mathrm{II}}, \Pi_{\mathrm{II}}^{5}$ & $2-1$ & 50 & 30 & -25 \\
\hline BVP $_{\mathrm{II}}, \Pi_{\mathrm{II}}^{6}$ & $2-1$ & 50 & 30 & 25 \\
\hline
\end{tabular}

Table 2. The functionals of the transient processes.

\begin{tabular}{|c|c|c|c|c|c|c|c|c|c|}
\hline Algorithm & Direction & $\tau_{\operatorname{Tr}}$ & $A_{\mathrm{Tr}}, 10^{3}$ & $M_{\mathrm{Tr}}^{i n}$ & $\overline{\bar{j}_{\mathrm{Tr}}^{i n}}$ & $M_{\mathrm{Tr}}^{\text {out }}$ & $\overline{j_{\text {Tr }}^{\text {out }}}$ & $Q_{\operatorname{Tr}}^{\text {in }}, 10^{3}$ & $Q_{\operatorname{Tr}}^{\text {out }}, 10^{3}$ \\
\hline $\mathrm{BVP}_{\mathrm{I}}, \Pi_{\mathrm{I}}^{1}$ & $1-2$ & 68.18 & 1.84 & 1.42 & 0.85 & 1.20 & 0.72 & 1.29 & 1.53 \\
\hline $\mathrm{BVP}_{\mathrm{I}}, \Pi_{\mathrm{I}}^{2}$ & $1-2$ & 53.84 & 1.51 & 1.18 & 0.90 & 0.95 & 0.72 & 1.28 & 1.59 \\
\hline $\mathrm{BVP}_{\mathrm{I}}, \Pi_{\mathrm{I}}^{3}$ & $2-1$ & 66.22 & 1.66 & 1.02 & 0.63 & 1.24 & 0.76 & 1.63 & 1.33 \\
\hline $\mathrm{BVP}_{\mathrm{I}}, \Pi_{\mathrm{I}}^{4}$ & $2-1$ & 49.43 & 1.21 & 0.71 & 0.58 & 0.94 & 0.77 & 1.71 & 1.29 \\
\hline $\mathrm{BVP}_{\mathrm{III}}, \Pi_{\mathrm{I}}^{1}$ & $1-2$ & 125.75 & 3.31 & 2.45 & 0.80 & 2.23 & 0.72 & 1.35 & 1.48 \\
\hline $\mathrm{BVP}_{\mathrm{III}}, \Pi_{\mathrm{III}}^{2}$ & $2-1$ & 123.50 & 3.11 & 2.06 & 0.68 & 2.28 & 0.75 & 1.51 & 1.37 \\
\hline BVP $_{I I}, \Pi_{I I I}^{1}$ & $1-2$ & 153.60 & 5.78 & 3.26 & 0.87 & 3.04 & 0.81 & 1.77 & 1.90 \\
\hline BVP $_{I I}, \Pi_{I I}^{2}$ & $1-2$ & 175.67 & 8.10 & 3.77 & 0.88 & 3.55 & 0.82 & 2.15 & 2.28 \\
\hline $\mathrm{BVP}_{\mathrm{II}}, \Pi_{\mathrm{II}}^{3}$ & $1-2$ & 93.39 & 2.66 & 1.38 & 0.85 & 1.70 & 0.75 & 1.39 & 1.56 \\
\hline $\mathrm{BVP}_{\mathrm{II}}, \Pi_{\mathrm{II}}^{4}$ & $2-1$ & 135.52 & 2.11 & 1.60 & 0.58 & 1.18 & 0.64 & 1.10 & 0.98 \\
\hline $\mathrm{BVP}_{\mathrm{II}}, \Pi_{\mathrm{II}}^{5}$ & $2-1$ & 169.56 & 2.33 & 2.35 & 0.57 & 1.80 & 0.62 & 0.99 & 0.91 \\
\hline BVP $_{\mathrm{II}}, \Pi_{\mathrm{II}}^{5}$ & $\overline{2-1}$ & 67.31 & 1.70 & 1.05 & 0.64 & 1.26 & 0.76 & 1.62 & 1.36 \\
\hline
\end{tabular}

\section{Conclusion}

Three mathematical models for the unsteady gas-dynamic processes of switching-over the pipeline from an actual stationary regime to another (target) stationary regime have been studied numerically in isothermal approximation. A boundary-value problem for the gas-dynamic equation system, describing the motion of the gas in the pipeline, corresponds to each model. The problems differ by the boundary functions, prescribed at the ends of the pipeline. In the problem $\mathrm{BVP}_{\mathrm{I}}$ for instance, dimensionless densities $\rho^{\text {in }}(\tau)$ and $\rho^{\text {out }}(\tau)$ are defined as the boundary data. In this case, we say, that the transient process realized in the correspondence by the model $\mathrm{BVP}_{\mathrm{I}}$, is controlled by these functions, acting at the pipeline's ends. In the model BVP $\mathrm{II}_{\mathrm{II}}$, they are functions $\rho^{i n}(\tau)$ at the inflow and dimensionless mass flow density $j^{\text {out }}(\tau)$ at the outflow, whereas in the model $\mathrm{BVP}_{\mathrm{I}}$ - the flow $j^{\text {in }}(\tau)$ and density $\rho^{i n}(\tau)$ are the control functions.

A unified model for the control functions was used in the study. The model restricts their class by the smooth functions, which monotonically vary from the value characteristic for the first stationary regime to another one specific for the target stationary regime. By the model, each control function $\psi(\tau)$ is defined by four real constants. They are two values, which $\psi(\tau)$ acquires in the first and second stationary regimes, and two parameters $\left(\tau_{\text {start }}^{\psi}\right.$ and $\left.\tau_{\text {end }}^{\psi}\right)$, defining the period of the function variation.

With the use of the control functions model, any transient process, realized by model $B V P_{K}, K=$ I, II, III is fully defined by a set $\Pi_{K}$ of eight parameters (four ones for each control function of the model $\left.B V P_{K}\right)$. Prescribing numerical values for the set $\Pi_{K}$ elements, we define an algorithm for control 
by a transient process realized in correspondence with the model $\mathrm{BVP}_{\mathrm{K}}$. The transient process realized by any algorithm can be characterized by several numerical parameters (functionals). They are the time duration $\tau_{T r}$, the work $\mathrm{A}_{\mathrm{Tr}}^{R}$ of viscous friction force executed during the process, the amounts $\mathrm{M}_{\mathrm{Tr}}^{i n}$ and $\mathrm{M}_{\mathrm{Tr}}^{\text {out }}$ of the gas entered into the pipeline at its inlet and delivered into the gas transmission system at the outlet, etc. The functionals allow evaluating some parameters of effectiveness of the transient process.

Results of case studies of twelve various algorithms of control by transient processes are presented in the paper. They are four algorithms for control model $B V P_{I}$, two ones for $B V P_{I I I}$, and six - for $B V P_{I I}$. The functionals were calculated for each case. The presented in Figs. 2-13 graphs make it possible to judge how the used model and parameters of control functions impact the transient processes. The data in Table 2, show that integral characteristics of transient process effectiveness depend on the chosen model and the algorithm of control by this process.

The mathematical models and results of the case studies, presented in the paper, can be used for development of strategy and practice for planning of transient regimes of pipeline operation. In particular, one can use them to check a planned transient process for its consistency with restrictions on the permissible values of the pressure and flow rate, to choose the algorithm, which provides a better efficiency of the pipeline, etc.

The class of control functions, used for modeling the transient process, can be extended by nonmonotonic functions and corresponding expanding the sets of control parameters $\Pi_{K}$. That will give new possibilities for transient processes controlling.

Involving into consideration a mathematical model for compressor stations will make it possible to use as the controls for transient processes the parameters determining regimes of their operation, namely - the power, developed by the compressors, their rotary speeds, consumed fuel, etc. Using the mathematical model, which determines among other parameters the gas temperature at compressor station's outflow, will enable considering the heat processes in the gas moving in the pipeline.

[1] Wang X., Economides M. Advanced natural gas engineering. Houston, Gulf Publishing Company (2013).

[2] Chaczykowski M. Sensitivity of pipeline gas flow model to the selection of the equation of state. Chemical engineering research and design. 87 (12), 1596-1603 (2009).

[3] Chaczykowski M. Transient flow in natural gas pipeline - the effect of pipeline thermal model. Applied Mathematical Modelling. 34 (4), 1051-1067, (2010).

[4] Oosterkamp A., Helgaker J. F., Ytrehus T. Modelling of natural gas pipe flow with rapid transients-case study of effect of ambient model. Energy Procedia. 64, 101-110 (2015).

[5] Farzaneh-Gord M., Rahbari M. R. Response of natural gas distribution pipeline networks to ambient temperature variation (unsteady simulation). Journal of Natural Gas Science and Engineering. 52, 94-105 (2018).

[6] Chekurin V., Khymko O. Mathematical modeling of a small pressure disturbance in gas flow of a long pipeline. Mathematical Modeling and Computing. 4 (2), 126-138 (2017).

[7] Chekurin V., Khymko O. Mathemetical model for propagation of long acoustic waves in the flow of a gas pipeline. Proceedings of XXII International IEEE Seminar/Workshop on direct and inverse problems of electromagnetic and acoustic wave theory (DIPED-2017). Dnipro, Ukraine. 80-83 (2017).

[8] Chekurin V., Khymko O. Wave of pressure in gas pipeline: a telegraph-type model. Proceedings of XXIII International IEEE Seminar/Workshop on Direct and Inverse Problems of Electromagnetic and Acoustic Wave Theory (DIPED-2018). Tbilisi, Georgia. 157-160 (2018).

[9] Chekurin V., Khymko O. Mathematical models for leak identification in a long gas pipeline. PhysicoMathematical modeling and Informational Technologies. 25, 157-169 (2017), (in Ukrainian).

[10] Chekurin V., Khymko O. Numerical study of transient processes in a long gas pipeline caused by depressurization. Physico-Mathematical modeling and Informational Technologies. 26, 100-111 (2017), (in Ukranian).

[11] Mathews J. H., Fink K. K. Numerical methods using Matlab. New Jersej, Prentice-Hall Publ. Inc. (2004).

Mathematical Modeling and Computing, Vol. 6, No. 2, pp. 220-238 (2019) 


\title{
Чисельне моделювання перехідних процесів у довгому газопроводі
}

\author{
Чекурін В. $\Phi^{1,2}$, Химко О. М. ${ }^{3}$ \\ ${ }^{1}$ Інститут прикладних проблем механіки і математики ім. Я. С. Підстригача НАН України, \\ вул. Наукова, 3-б, Львів, 79060, Україна \\ 2 Куявсъко-Поморсъкий Університет у Бидгощі, \\ вул. Торунъсъка, 55-57, Бидгощ, 85-023, Польща \\ ${ }^{3}$ Національний університет "Львівсъка політехніка", \\ вул. С. Бандери, 12, Львів, 79013, Украӥна
}

У статті розглянуто нестаціонарні процеси ізотермічної течії газу, що виникають у довгому трубопроводі під час його переходу з одного стаціонарного режиму на інший. Дослідження проведено з використанням одновимірної моделі динаміки газу, в яку входять рівняння збереження маси та імпульсу газу, записані відносно безрозмірних густин маси та потоку. Для цієї системи сформульовано три крайові задачі, які визначають три моделі керування перехідними процесами. Задачі відрізняються граничними функціями, заданими на кінцях трубопроводу. Запропоновано уніфіковану модель для функцій керування. За цією моделлю будь-яка функція керування визначається чотирма дійсними параметрами. Виконано чисельний аналіз перехідних процесів, з використанням різних моделей керування за різних значень параметрів керування. У статті розглянуто деякі можливості застосування таких математичних моделей та результати проведених числових досліджень для планування перехідних режимів роботи магістральних газопроводів.

Ключові слова: газова динаміка, довгий трубопровід, перехідні прощеси.

2000 MSC: 76D55

Удк: 533:519.6:621.64.029 